\title{
Open clusters
}

\section{Fundamental parameters of B stars in Collinder 223, Hogg 16, NGC 2645, NGC 3114, and NGC 6025}

\author{
Y. Aidelman ${ }^{1,2, \star \star}$, L. S. Cidale ${ }^{1,2, \star \star \star}$, J. Zorec ${ }^{3,4}$, and J. A. Panei ${ }^{1,2, \star \star \star}$ \\ ${ }^{1}$ Departamento de Espectroscopía, Facultad de Ciencias Astronómicas y Geofísicas, Universidad Nacional de La Plata (UNLP), \\ Paseo del Bosque S/N, 1900 La Plata, Argentina \\ e-mail: aidelman@fcaglp.unlp.edu.ar \\ 2 Instituto de Astrofísica La Plata, CCT La Plata, CONICET-UNLP, Paseo del Bosque S/N, 1900 La Plata, Argentina \\ 3 Sorbonne Université, UPMC Univ. Paris 6, 934 UMR7095-IAP, 75014 Paris, France \\ 4 CNRS, 934 UMR7095-IAP, Institut d'Astrophysique de Paris, 98bis Bd. Arago, 75014 Paris, France
}

Received 30 September 2014 / Accepted 28 January 2015

\begin{abstract}
Context. The knowledge of accurate values of effective temperature, surface gravity, and luminosity of stars in open clusters is very important not only to derive cluster distances and ages but also to discuss the stellar structure and evolution. Unfortunately, stellar parameters are still very scarce.

Aims. Our goal is to study five open clusters to derive stellar parameters of the B and Be star population and discuss the cluster properties. In a near future, we intend to gather a statistically relevant samples of Be stars to discuss their origin and evolution.

Methods. We use the Barbier-Chalonge-Divan spectrophotometric system, based on the study of low-resolution spectra around the Balmer discontinuity, since it is independent of the interstellar and circumstellar extinction and provides accurate Hertzsprung-Russell diagrams and stellar parameters.

Results. We determine stellar fundamental parameters, such as effective temperatures, surface gravities, spectral types, luminosity classes, absolute and bolometric magnitudes and colour gradient excesses of the stars in the field of Collinder 223, Hogg 16, NGC 2645, NGC 3114, and NGC 6025. Additional information, mainly masses and ages of cluster stellar populations, is obtained using stellar evolution models. In most cases, stellar fundamental parameters have been derived for the first time. We also discuss the derived cluster properties of reddening, age and distance.

Conclusions. Collinder 223 cluster parameters are $\overline{E(B-V)}=0.25 \pm 0.03$ mag and $\overline{\left(m_{\mathrm{v}}-M_{\mathrm{v}}\right)_{0}}=11.21 \pm 0.25 \mathrm{mag}$. In Hogg 16 , we clearly distinguish two groups of stars (Hogg 16a and Hogg 16b) with very different mean true distance moduli (8.91 $\pm 0.26 \mathrm{mag}$ and $12.51 \pm 0.38 \mathrm{mag})$, mean colour excesses $(0.26 \pm 0.03 \mathrm{mag}$ and $0.63 \pm 0.08 \mathrm{mag})$, and spectral types (B early-type and B late-/Atype stars, respectively). The farthest group could be merged with Collinder 272. NGC 2645 is a young cluster $(<14 \mathrm{Myr})$ with $\overline{E(B-V)}=0.58 \pm 0.05 \mathrm{mag}$ and $\overline{\left(m_{\mathrm{v}}-M_{\mathrm{v}}\right)_{0}}=12.18 \pm 0.30 \mathrm{mag}$. The cluster parameters of NGC 3114 are $\overline{E(B-V)}=0.10 \pm 0.01 \mathrm{mag}$ and $\overline{\left(m_{\mathrm{v}}-M_{\mathrm{v}}\right)_{0}}=9.20 \pm 0.15 \mathrm{mag}$. This cluster presents an important population of Be star, but it is difficult to define the cluster membership of stars because of the high contamination by field stars or the possible overlapping with a nearby cluster. Finally, we derive the following cluster parameters of NGC 6025: $\overline{E(B-V)}=0.34 \pm 0.02 \mathrm{mag}, \overline{\left(m_{\mathrm{v}}-M_{\mathrm{v}}\right)_{0}}=9.25 \pm 0.17 \mathrm{mag}$, and an age between $40 \mathrm{Myr}$ and $69 \mathrm{Myr}$. In all the cases, new Be candidate stars are reported based on the appearance of a second Balmer discontinuity.
\end{abstract}

Key words. open clusters and associations: individual: Collinder 223 - stars: fundamental parameters - stars: emission-line, Be open clusters and associations: individual: Hogg 16 - open clusters and associations: individual: NGC 2645 -

open clusters and associations: individual: NGC 3114

\section{Introduction}

Open clusters represent samples of stars that are thought to be coeval, share a common origin, and hence have the same chemical composition. These properties make open clusters ideal targets to study the stellar structure and evolution. In addition,

\footnotetext{
* Observations taken at CASLEO, operating under agreement of CONICET and the Universities of La Plata, Córdoba and San Juan, Argentina.

$\star \star$ Fellow of CONICET, Argentina.

$\star \star \star$ Member of the Carrera del Investigador Científico, CONICET, Argentina.
}

because of their compact shapes, they are often used as distance calibrators and galactic spiral arm tracers. These results, together with metallicity determinations and kinematic studies of the Galaxy, enable us to trace the history of the galaxy formation.

Most of the parameters that describe the open clusters are based on photometric studies since the stars that comprise them have scarce spectroscopic data. However, the knowledge of the fundamental parameters of the stars (spectral type, luminosity class, effective temperature, surface gravity, etc.) are very important not only to determine the cluster distance and age, but also to analyse and discuss the formation and evolution of stars with peculiarities, such as Be stars and chemically peculiar stars, 
and the incidence of binarity. In a recent work, Aidelman et al. (2012, hereinafter Paper I) introduced the spectrophotometric Barbier-Chalonge-Divan (BCD) method (Barbier \& Chalonge 1941; Chalonge \& Divan 1952) as a tool to evaluate accurate physical parameters of the cluster's stars and the cluster properties in a fast and direct way. On the other hand, as the BCD parameters are free of interstellar and circumstellar absorption, the location of stars in the Hertzsprung-Russell (HR) diagram is more accurate than classical methods based on plain photometry, thus allowing for better determinations of cluster ages. Particularly, the BCD method also enables us to isolate each star in very crowded stellar regions and discuss its cluster membership taking its colour excess and distance estimate into account.

This work presents a spectrophotometric investigation to derive stellar parameters of around 110 B-type stars in the field of the galactic clusters Collinder 223, Hogg 16, NGC 2645, NGC 3114, and NGC 6025. Some of them are located in the fourth galactic quadrant towards the Carina arm tangent, which makes a proper distance estimate difficult. This geometrical projection favours overlapping clusters and makes it difficult to set a clear membership identification.

The paper is organized as follow: data acquisition and reduction procedure are given in Sect. 2. Spectrophotometric results on the cluster parameters and memberships are provided in Sect. 3. A discussion about the methodology used and results obtained are also presented. Possible scenarios linked to the observed negative gradient excess in NGC 3114 are delineated in Sect. 4. The conclusions are summarized in Sect. 5.

\section{Observations}

Low-resolution spectra of stars in several open clusters were obtained in different observing runs between the years 2002 and 2013. Two of these clusters were published in Paper I and here we present the results corresponding to other five clusters.

We performed the observations at the Complejo Astronómico El Leoncito (CASLEO), San Juan, Argentina, using the Boller \& Chivens spectrograph attached to the Sahade $2.15 \mathrm{~m}$ telescope. All the spectra were taken with the $6001 \mathrm{~mm}^{-1}$ grating (\#80). Before 2011, the covered spectral wavelength was 3500-4700 $\AA$, while the latest observations were carried out in the wavelength interval 3500-5000 $\AA$. The effective spectral resolution obtained is $4.53 \AA$ every two pixels.

We took the observations at the lowest possible zenith distance to minimize refraction effects due to the Earth's atmosphere and, thus, to avoid light losses as a function of wavelength. We also used the widest possible opening of the slit to minimize light losses. Each night we observed several flux standard stars to perform the spectral flux calibrations.

The reduction procedure was carried out with the IRAF $^{1}$ software package (version 2.14.1) and all spectra were corrected for atmospheric extinction.

\section{Results}

To analyse the spectroscopic data of the population of $B$ and Be stars in open clusters, we used the BCD spectrophotometric

\footnotetext{
1 IRAF is distributed by the National Optical Astronomy Observatory, which is operated by the Association of Universities for Research in Astronomy (AURA), Inc., under cooperative agreement with the National Science Foundation.
}

method. This method is based on the measurement of four parameters on low-resolution spectra around the Balmer discontinuity. Two of them, $D$ and $\lambda_{1}$, describe the height and the average spectral position of the Balmer jump, respectively. The third and fourth parameters are the colour gradients, $\Phi_{\mathrm{uv}}$ and $\Phi_{\mathrm{b}}$, which give the slope of the Balmer and Paschen continua, respectively. All these parameters lead to direct determinations of the star's effective temperature, $T_{\text {eff }}$, logarithm of the surface gravity, $\log g$, absolute visual magnitude, $M_{\mathrm{v}}$, and bolometric magnitude, $M_{\mathrm{bol}}$, as is described in Paper I.

It was shown that the appearance of a second Balmer discontinuity, near $\lambda 3648 \AA$, in emission or in absorption, is correlated with the presence of Balmer emission lines (Divan et al. 1982; Moujtahid et al. 2000). Since our spectra do not enable us to see either the $\mathrm{H} \alpha$ line, which is out of the spectral range, or the possible weak emission components in the $\mathrm{H} \beta$ line, because of the low resolution of our spectra, the presence of a second Balmer discontinuity provides justification that the star is a Balmer emission-line object.

For each cluster, the result of their individual members are gathered in two tables. The first table shows the star identification, the observed BCD parameters $D, \lambda_{1}, \Phi_{\mathrm{b}}$ and the stellar physical properties (spectral type and luminosity class, $T_{\text {eff }}$, $\log g, M_{\mathrm{v}}, M_{\mathrm{bol}}$, and the intrinsic colour gradient $\left.\Phi_{\mathrm{b}}^{0}\right)$. Regarding the spectral types derived from the BCD method, when possible, we have tested them with those of the standard MK system. In some occasions, we were not able to interpolate or extrapolate the BCD parameters into the BCD-calibration tables. In those cases, the MK-classification system was used, while the fundamental parameters were taken from Cox (2000) and the intrinsic colour gradients were derived using the relation between $\Phi_{\mathrm{b}}$ and $(B-V)$ given by Moujtahid et al. (1998).

The second table includes for each star: the visual apparent magnitude $m_{\mathrm{v}}$, the obtained colour excess $E(B-V)$, the true distance modulus $\left(m_{\mathrm{v}}-M_{\mathrm{v}}\right)_{0}$, the distance $d$, the luminosity $\mathcal{L}$ relative to the solar one, the stellar mass $M$, and age. Stellar masses were determined by interpolation using non-rotating evolutionary models with $Z=0.014$, given by Ekström et al. (2012).

For each cluster, we estimate the arithmetic mean distance modulus, reddening, and age, with their corresponding standard errors $^{2}$, using data of a pre-selected sample of stars assumed to be probable cluster members $(\mathrm{pm})$. To determine the star membership, we consider here a more restrictive criterion than that proposed in Paper I. That is, when the star's distance modulus is outside the region defined by three times the standard deviation, $\sigma_{N-1}$, the star is considered a non-member (nm) of the cluster. If the star falls inside a region between $2 \sigma_{N-1}$ and $3 \sigma_{N-1}$, the star is considered a probable non-member $(p n m)$. Finally, the star is assumed to be a member when it is inside a region of $2 \sigma_{N-1}$ size around the mean cluster distance modulus.

The initial sample of probable star members is roughly preselected by constructing a histogram of frequencies of stellar distance-moduli with classes chosen according to Sturge's rule $^{3}$. This histogram is corrected for errors affecting the individual stellar distance-modulus determination using Gaussian kernel estimators (Bowman \& Azzalini 1997), which produced a smoothed distribution of frequencies. We adopted the weighted mean value for the distance modulus and colour excess as a rep-

\footnotetext{
The standard error, $\epsilon=\sigma_{N-1} / \sqrt{N}$, is defined as the standard deviation for a finite population, $\sigma_{N-1}$, divided by the square root of the sample size, $N$.

3 http://www . jstor. org/stable/2965501
} 
Table 1. Mean properties of open clusters obtained from the BCD method.

\begin{tabular}{lcrcc}
\hline \hline Cluster & $\begin{array}{c}\overline{E(B-V)} \\
{[\mathrm{mag}]}\end{array}$ & $\begin{array}{c}\overline{\left(m_{\mathrm{v}}-M_{\mathrm{v}}\right)_{0}} \\
{[\mathrm{mag}]}\end{array}$ & $\begin{array}{c}\langle d\rangle \\
{[\mathrm{pc}]}\end{array}$ & $\begin{array}{c}\text { Age } \\
{[\mathrm{Myr}]}\end{array}$ \\
\hline Cr 223 & $0.25 \pm 0.03$ & $11.21 \pm 0.25$ & $1746_{-186}^{+209}$ & $\sim 25$ \\
Hogg 16a & $0.26 \pm 0.03$ & $8.91 \pm 0.26$ & $605_{-69}^{+77}$ & $<40$ \\
Hogg 16b & $0.63 \pm 0.08$ & $12.51 \pm 0.38$ & $3172_{-515}^{+614}$ & $11 \pm 3$ \\
NGC 2645 & $0.58 \pm 0.05$ & $12.18 \pm 0.30$ & $2726_{-354}^{+407}$ & $6-10$ \\
NGC 3114 & $0.10 \pm 0.01$ & $9.20 \pm 0.15$ & $694_{-46}^{+49}$ & $\ldots$ \\
NGC 6025 & $0.34 \pm 0.02$ & $9.25 \pm 0.17$ & $709_{-53}^{+57}$ & $68 \pm 13$ \\
\hline
\end{tabular}

resentative quantity for the cluster. The weighted mean is estimated using the entire continuous distribution over the interval indicated by the histogram. The adopted uncertainties of each of these quantities are the weighted standard deviations (Smart 1958), $\sigma_{\mathrm{w}}$, which are slightly larger than the arithmetic standard deviation. As the expected cluster size is about a few tens of pcs, only stars inside one $\sigma_{\mathrm{w}}$ of the mean distance modulus are considered probable members of the cluster (see results in Sects. 3.1-3.5).

Finally, once the cluster sample is defined, we simply use the arithmetic mean values and standard errors to estimate the cluster parameters. The final list of star members is made with the criterion suggested at the beginning of this section.

The mean cluster age is derived in two different ways: the first is by fitting isochrones selected from Ekström et al. (2012), the second is determined by calculating the average of the age of individual star members (for details see Paper I). In the table, we list the age obtained by the ZAMS fitting technique only when the discrepancy between both methods is large. The obtained parameters of the clusters are summarized in Table 1.

\subsection{Collinder223}

Collinder $223\left(\mathrm{Cr} 223, \alpha=10^{\mathrm{h}} 32^{\mathrm{m}} 16^{\mathrm{s}}\right.$ and $\delta=-60^{\circ} 01^{\mathrm{m}} \cdot 2$; 2000 ) is a poorly studied medium-size open cluster located in the Carina region, where the Carina spiral feature is seen tangentially (cf. Claria \& Lapasset 1991). The $U B V$ photometric data have only been obtained by Claria \& Lapasset (1991), who showed that Collinder 223 is a genuine open cluster of only B-type stars. Its distance and age are quite uncertain, ranging from $d=1686 \mathrm{pc}$ and $t=257 \mathrm{Myr}$ (Kharchenko et al. 2005) to $d=2900 \pm 270 \mathrm{pc}$ and $t=36 \mathrm{Myr}$ (Claria \& Lapasset 1991). Other estimates indicate $d=2820 \pm 190$ and $t=100 \mathrm{Myr}$ (Tadross 2004), and $d=2130$ pc (Rastorguev et al. 1999, who based their results on the statistical-parallax method with proper motions reduced to the HIPPARCOS reference system). Colour excess determinations by Claria \& Lapasset (1991) and Tadross (2004) show agreement (0.26 mag and $0.25 \mathrm{mag}$, respectively).

Apart from the brightest stars with known spectral types and luminosity classes, none of the spectroscopic studies devoted to this cluster perform the spectral classification. Among the stars with previously known spectral types, we point out No. 80 and HD 305296 which are classified as B2Ve by Graham (1970), and stars Nos. 2 and 4, listed in the catalogue of Houk \& Cowley (1975), which are classified as B9.5IV/V and B8III, respectively. The latter, star No. 4, was also classified by Wade \& Smith (1985) as B5/6, B6, and B3, in three different ways using the $u v b y \mathrm{H} \beta$ photometry and the ultraviolet flux. Other stars listed in
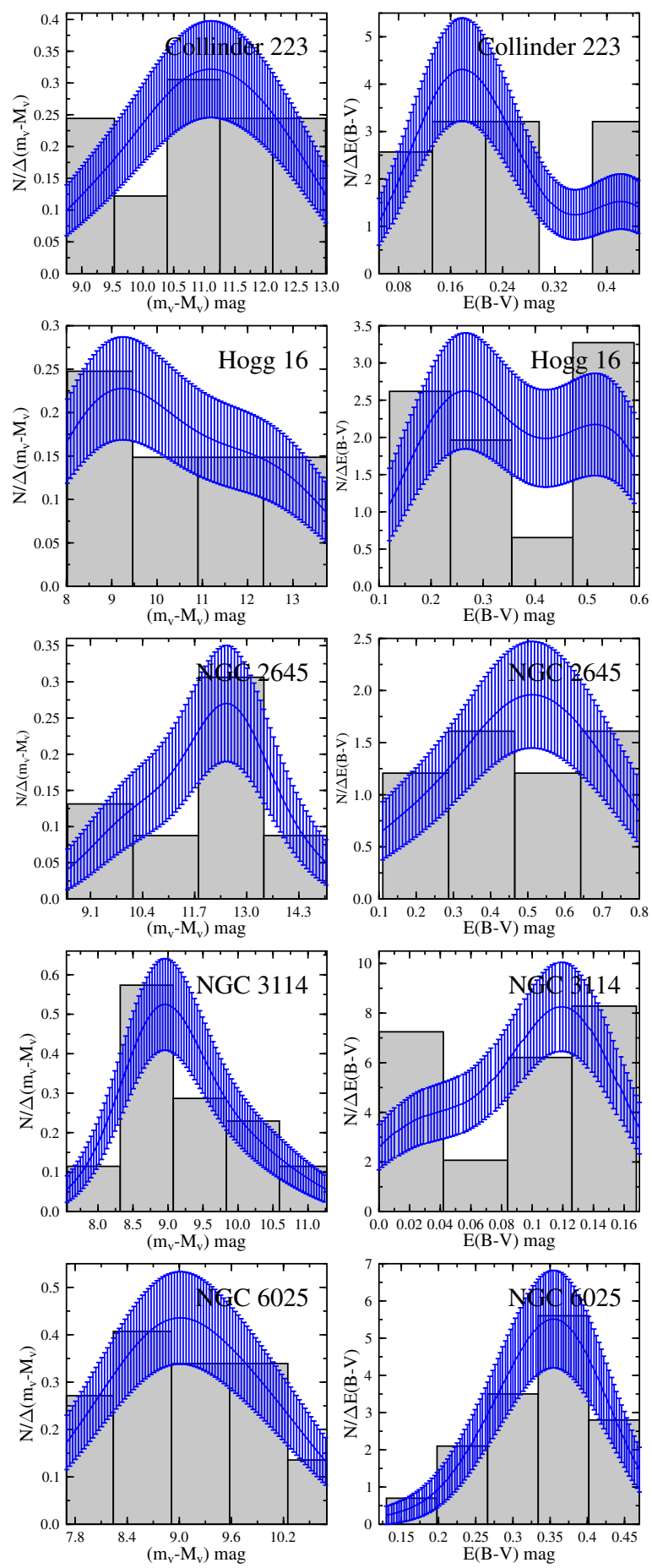

Fig. 1. Frequency distribution of stars (left) and colour excesses (right) per interval of distance moduli (left). Probable members are expected inside a spatial volume of twice the standard deviation.

the catalogue of Nesterov et al. (1995): Nos. 9, 29, 48, 56, and 57 are listed as A0; No. 20 as A; No. 106 as F2; HD 305291 as B2.

Because of the lack of a homogeneous classification, we made use of the BCD method to derive the fundamental parameters of many stars in the field of $\mathrm{Cr} 223$. These results are shown in Tables 5 and 6 . Based on the histogram of frequencies of stellar distance-moduli, we selected a first sample of probable cluster members (see Fig. 1, upper panel). From this pre-sample, the BCD absolute magnitudes and colour excesses enable us to obtain the mean cluster 
Table 2. NGC 3114: Spectral classification done in previous works. Nomenclature according to Lyngå (1962).

\begin{tabular}{|c|c|c|c|}
\hline $\begin{array}{c}\text { ID } \\
\text { Lynga }\end{array}$ & $\begin{array}{l}\text { Other } \\
\text { designation }\end{array}$ & $\begin{array}{l}\text { Spectral } \\
\text { types }\end{array}$ & References \\
\hline 003 & HD 87458 & B8III, B8II/III, B8III, B8IIIe & $1,2,3,10$ \\
\hline 004 & HD 87241 & B9III, B9III, B8.5IVp (Si II), B8.5, B8.5IIIe, B9 & $1,2,3,8,10,11$ \\
\hline 005 & HD 87266 & B1V, B3IV, B2IV/Vp, B2.5V, B9 (Si ) & $1,2,3,10,12$ \\
\hline 006 & HD 87137 & B8II/III, B9IV, B8III & $2,3,10$ \\
\hline 007 & HD 87042 & B9V, B9.5IV/V, B9III/IV, B9, B9.5V & $1,2,3,8,10$ \\
\hline 009 & HD 87222 & B3V, B3IV, B2IVp, B3V, B3, O & $1,2,3,10,11$ \\
\hline 010 & HD 87349 & B8IV, B8V, B8III, B8, B7V & $1,2,3,8,10$ \\
\hline 011 & HD 87031 & B9IV, B9III, B9.5IV, B9III & $1,2,3,10$ \\
\hline 013 & HD 304848 & B9IV, B9 & 3,9 \\
\hline 015 & HD 87612 & B7V, B8V, B4Vn, B6V & $1,2,3,10$ \\
\hline 028 & HD 87404 & B8IV, B9, B7Vn, B8IV & $1,2,3,6$ \\
\hline 033 & HD 87818 & B8/B9IV, A0Vn & 2,3 \\
\hline 040 & HD 304894 & B9V, A0 & 1,9 \\
\hline 047 & HD 87565 & B4V, B4IV, B2Vnne, B4IV, B4V, B2Ve & $1,2,3,5,6,10$ \\
\hline 066 & HD 304841 & A, B9 & 9,12 \\
\hline 067 & HD 304840 & A & 9 \\
\hline 079 & HD 304829 & $\mathrm{~A} 3, \mathrm{~A} 2$ & 4,9 \\
\hline 085 & CPD-59 1748 & B9IV: & 1 \\
\hline 088 & HD 304837 & B9 & 9 \\
\hline 091 & HD 87125 & B9IV, B9V, B8.5III shell & $1,2,10$ \\
\hline 097 & HD 87205 & B9V, B9II/III & 1,2 \\
\hline 099 & CPD-59 1677 & $\cdots$ & \\
\hline 100 & HD 304836 & B6/B9 & 4 \\
\hline 102 & HD 304835 & A, B9.5III & 9,10 \\
\hline 105 & CPD-59 1666 & $\cdots$ & \\
\hline 124 & HD 304854 & B9, A & 4,9 \\
\hline 126 & HD 87309 & B8V: & 1 \\
\hline 127 & HD 304855 & A & 9 \\
\hline 129 & HD 87366 & B9V:, B9III, B9V & $1,5,6$ \\
\hline 130 & HD 304856 & A & 9 \\
\hline 132 & HD 304831 & A0 & 9 \\
\hline 134 & HD 87567 & A & 2 \\
\hline 136 & HD 87581 & B8/B9II/III & 2 \\
\hline 141 & HD 304832 & A0, B3III & 9,10 \\
\hline 147 & HD 304858 & A & 9 \\
\hline 149 & HD 87507 & B9III, B9II/III, B8III/IV & $1,2,10$ \\
\hline 186 & HD 86981 & B9IV, B8/B9II/III & 1,2 \\
\hline$\cdots$ & HD 87436 & A7III, A6II/III, A6II, A6II, A4 & $1,2,7,10,11$ \\
\hline$\cdots$ & HD 87801 & B4V, B2/B3Vn, B4.5IV & $1,2,10$ \\
\hline$\cdots$ & HD 304838 & $\mathrm{~B} 6 / \mathrm{B} 9, \mathrm{~A} 0$ & 4,9 \\
\hline$\cdots$ & HD 304839 & B6/B8, B9 & 4,9 \\
\hline$\cdots$ & CPD-59 1691 & $\cdots$ & \\
\hline$\cdots$ & J10015881-6001125 & $\cdots$ & \\
\hline
\end{tabular}

References. (1) Frye et al. (1970); (2) Houk \& Cowley (1975); (3) Levato \& Malaroda (1975); (4) Amieux \& Burnage (1981); (5) Jaschek \& Egret (1982), (6) Mermilliod (1982a); (7) Gray \& Garrison (1989); (8) Hamdy et al. (1993); (9) Nesterov et al. (1995); (10) González \& Lapasset (2001); (11) Renson \& Manfroid (2009); and (12) Bychkov et al. (2009).

parameters: $\overline{E(B-V)}=0.25 \pm 0.03 \mathrm{mag}, \overline{\left(m_{\mathrm{v}}-M_{\mathrm{v}}\right)_{0}}=11.21 \pm$ $0.25 \mathrm{mag}$ and $\bar{d}=1990 \pm 220 \mathrm{pc}$. All values are based on the average of the data for members and $\mathrm{pnm}$. The average of individual distances is only slightly larger than the value obtained using the Pogson law and the mean distance modulus, $\langle d\rangle=1746 \mathrm{pc}$.

To determine the cluster age, we constructed an HR diagram. Using the isochrones given by Ekström et al. (2012) and considering the location of the most evolved stars, HD 305291 and HD 305295 , both classified as pnm, we estimated their age of about 25 Myr (see Fig. 3). Instead, if we use the average of ages computed from the individual stellar ages (members and pnm) we obtain $136 \pm 32$ Myr. However, this last value could be overestimated because of possible anomalies in the colour excess, as is discussed in Sect. 4. Therefore, the age of the cluster is still uncertain and a larger number of objects is needed to obtain a more reliable age determination.
Our distance determination agrees with the value obtained by Kharchenko et al. (2005, 1686 pc). The colour excess derived with the BCD method $(0.25 \mathrm{mag})$ agrees very well with the estimates done by Claria \& Lapasset $(1991, E(B-V)=0.26 \pm$ $0.03 \mathrm{mag})$ and Tadross $(2004, E(B-V)=0.25 \pm 0.015 \mathrm{mag})$.

Then considering the standard error listed in Table 1 and our criterion to define the cluster membership (see Sect. 3), we find that stars Nos. 3, 20, 48, 49, 56, 57, 58, 105, and 106 are members. Among these objects, stars Nos. 48, 49, 57, and 58 were considered by Claria \& Lapasset (1991) as only possible members of the cluster. Stars Nos. 5, 9, 21, and 35, HD 305291 and HD 305296 are pnm, while stars Nos. 2, 4, 29, and 80 are definitely non-members.

The obtained BCD spectral types agree very well with previous determinations except for the stars Nos. 4 and 106. In the case of No. 4 we agree with the classification done by 
Table 3. NGC 3114: Stars with anomalous colour gradients.

\begin{tabular}{clccc}
\hline \hline $\begin{array}{c}\text { ID } \\
\text { Lynga }\end{array}$ & $\begin{array}{l}\text { Other } \\
\text { designation }\end{array}$ & $\begin{array}{c}\Phi_{\mathrm{b}} \\
{[\mu]}\end{array}$ & $\begin{array}{c}\Phi_{\mathrm{b}}^{0} \\
{[\mu]}\end{array}$ & $\left(\Phi_{\mathrm{b}}-\Phi_{\mathrm{b}}^{0}\right)$ \\
\hline 003 & HD 87 458 & 0.42 & $0.78 \pm 0.01$ & -0.36 \\
005 & HD 87 266 & 0.62 & $0.72 \pm 0.01$ & -0.10 \\
006 & HD 87 137dd & 0.73 & $0.85 \pm 0.02$ & -0.12 \\
007 & HD 87 042 & 0.78 & $1.07 \pm 0.02$ & -0.29 \\
010 & HD 87 349 & 0.22 & $0.81 \pm 0.01$ & -0.59 \\
013 & HD 304 848 & 0.77 & $0.81 \pm 0.01$ & -0.04 \\
015 & HD 87 612 & 0.66 & $0.75 \pm 0.01$ & -0.10 \\
028 & HD 87 404 & 0.70 & $0.79 \pm 0.01$ & -0.09 \\
040 & HD 304 894 & 0.25 & $0.95 \pm 0.04$ & -0.70 \\
047 & HD 87 565 & 0.30 & $0.75 \pm 0.01$ & -0.45 \\
085 & CPD 591748 & 0.34 & $1.07 \pm 0.05$ & -0.73 \\
088 & HD 304 837 & 0.85 & $1.02 \pm 0.05$ & -0.17 \\
097 & HD 87 205 & 0.79 & $0.85 \pm 0.02$ & -0.06 \\
129 & HD 87 366 & 0.68 & $0.83 \pm 0.02$ & -0.15 \\
186 & HD 86 981 & 0.73 & $0.81 \pm 0.02$ & -0.08 \\
$\cdots$ & HD 87 436 & 1.30 & $1.79 \pm 0.02$ & -0.49 \\
$\cdots$ & HD 87 801 & 0.67 & $0.71 \pm 0.00$ & -0.04 \\
$\cdots$ & J10015881-6001125 & 0.60 & $0.88 \pm 0.02$ & -0.28 \\
\hline
\end{tabular}

Notes. Nomenclature according to Lyngå (1962). ${ }^{(d d)}$ Stars with double Balmer discontinuity.

Wade \& Smith (1985) based on the $u v b y \mathrm{H} \beta$ photometry and UV fluxes. For No. 106, we confirmed our BCD classification with the MK method and find that this star is a B5 IV and not a F-type star. We have determined the spectral types of the seven remaining stars of our sample for the first time.

In addition, we also find four stars with a second Balmer discontinuity (Nos. 2, 4, 21, and 106), which indicates the presence of a circumstellar envelope, although no emission in the $\mathrm{H} \alpha$ line is reported in the literature.

On the other hand, this cluster has two stars reported as blue stragglers: Nos. 80 and 35 (Claria \& Lapasset 1991). However, as we mentioned previously, No. 80 is a B0V-type star, located beyond the cluster $(d=3936 \pm 1177 \mathrm{pc})$, and No. 35, according to the BCD method, is a B3II probable non-member object. Instead, if we assume that the age of the cluster is $136 \mathrm{Myr}$ (average age obtained from individual stellar values), the BCD classification suggests that HD 305296 could be a B0V-type blue straggler star.

\section{2. $\operatorname{Hogg} 16$}

$\operatorname{Hogg} 16\left(\alpha=13^{\mathrm{h}} 29^{\mathrm{m}} 18^{\mathrm{s}}\right.$ and $\left.\delta=-61^{\circ} 12^{\mathrm{m}} \cdot 0 ; 2000\right)$ is an open cluster located in the constellation of Centaurus. Hogg (1965) suggested that Hogg 16 could be merged with Collinder $272^{4}$. Considering the close angular distance between both clusters, only $10^{\mathrm{m}}$, Vazquez \& Feinstein (1991) support this idea, while Vazquez et al. (1997) suggest that these clusters could be evolutionarily linked.

A photometric study was performed by several authors: Moffat \& Vogt (1973b) determined $E(B-V)=0.42 \mathrm{mag}$, $\left(m_{\mathrm{v}}-M_{\mathrm{v}}\right)_{0}=12.64 \mathrm{mag}(d=1.81 \mathrm{kpc})$; Fenkart et al. (1977) derived a distance modulus of $8.90 \mathrm{mag}(d=603 \mathrm{pc})$ and an age <510 Myr; Vazquez \& Feinstein (1991) determined $E(B-V)=0.44 \mathrm{mag},\left(m_{\mathrm{v}}-M_{\mathrm{v}}\right)_{0}=11.65 \mathrm{mag}(d=2130 \mathrm{pc})$ and $t=25.8 \pm 1.5$ Myr. Instead, Kharchenko et al. (2005) derived

\footnotetext{
4 In the original work the author wrote Collinder 227 but as the coordinates of this cluster are very different we assumed it was a typographic mistake.
}

a distance of $1.585 \mathrm{kpc}$, which is similar to the HIPPARCOS distance estimate, $1.90 \mathrm{kpc}$, Rastorguev et al. (1999), and $\log t=$ 7.26 ( $\sim 18$ Myr). Later, Kharchenko et al. (2009b) determined $E(B-V)=0.41 \mathrm{mag}$.

With respect to previous spectral classifications, we found that stars Nos. 9, 50, 52, and 68 appear in the catalogue of Houk \& Cowley (1975) as A, B9V, B8/9III, and B8V, respectively. Five stars of our sample were classified by Fitzgerald et al. (1979a) as follows: No. 1 as B2III, No. 2 as B2V, No. 3 as B3V, No. 6 as B1IV and No. 9 as B2e. However, star No. 3 was also classified by Garrison et al. (1977) as B1.5V, while No. 68 was classified as a B7/8-type (Vazquez \& Feinstein 1991).

From either the BCD system and the histograms of frequencies (Fig. 1, second line of panels), we clearly distinguish two populations of stars showing very different distance moduli, colour excesses and spectral types (see also Tables 7 and 8). One group consists mainly of late B- and A-type stars (hereinafter Hogg 16a) and is located at a mean true distance modulus of $8.91 \pm 0.26 \mathrm{mag}$, which corresponds to a distance of $605 \mathrm{pc}$. This distance is similar to the average distance obtained using individual values, $\bar{d}=610 \pm 70 \mathrm{pc}$. The mean colour excess, $\overline{E(B-V)}=0.26 \pm 0.03 \mathrm{mag}$, was derived by averaging the values obtained for stars Nos. 5, 14, 49, 50, 52, 68, 74, and 75. The other group is comprised of early B-type stars (hereinafter Hogg 16b), characterized by a high reddening located at $\left(m_{\mathrm{v}}-M_{\mathrm{v}}\right)_{0}=12.51 \pm 0.38 \mathrm{mag}$, which corresponds to a distance of $3172 \mathrm{pc}$. While the average distance and colour excess are $\bar{d}=3420 \pm 590 \mathrm{pc}$ and $\overline{E(B-V)}=0.63 \pm 0.08 \mathrm{mag}$, respectively, if we adopt the average values derived with stars Nos. 1, $2,3,6,9$, and 53. This result confirms the apparent visual overlapping of the two different cluster system along the line of sight.

With the BCD data we plot an HR diagram (see Fig. 4). This diagram shows that the age of Hogg $16 \mathrm{a}$ is $<40 \mathrm{Myr}$, while the age of Hogg 16b is between $10 \mathrm{Myr}$ and $16 \mathrm{Myr}$. On the other hand, the averages of the individual members yield 233 $\pm 62 \mathrm{Myr}$ and $11 \pm 3 \mathrm{Myr}$, respectively. The age of the first group is more uncertain probably because of the lack of a significant sample of objects.

The BCD cluster parameters of Hogg 16a agrees very well with the $\left(m_{\mathrm{v}}-M_{\mathrm{v}}\right)_{0}$ determined by Fenkart et al. (1977, $8.90 \mathrm{mag}$ ), but our colour excesses are smaller/greater than the values calculated by Moffat \& Vogt (1973b, $0.42 \mathrm{mag}$ ) or Vazquez \& Feinstein (1991, 0.44 mag).

According to Vazquez \& Feinstein (1991) and Vazquez et al. (1997) stars Nos. 9 and 53 are non-members of Hogg 16. The latter, star No. 53, probably belongs to Collinder 272 . However, considering the distance and age we estimated for Hogg 16b, this more distant group of stars could be merged with Collinder 272, whose estimated distance is uncertain, between 2045 pc and 4018 pc (Kharchenko et al. 2005; Pandey et al. 2010, respectively) and age $\log t=7.227^{5}$.

From the whole sample that we studied, we reported the spectral types of five stars for the first time. In general, we derived spectral types that are earlier than those reported in previous works. Among the known population of Be stars in Hogg 16, two of them (stars Nos. 3 and 68) show a second Balmer jump.

\subsection{NGC 2645}

NGC 2645 (Pismis 6; $\alpha=08^{\mathrm{h}} 39^{\mathrm{m}} 03^{\mathrm{s}}$ and $\delta=-46^{\circ} 14 .^{\mathrm{m}} 0$; 2000) is a small galactic cluster located in the Vela region.

5 See WEBDA database http://webda. physics . muni.cz 
Table 4. NGC 6025: Spectral classification done in previous works.

\begin{tabular}{clll}
\hline \hline $\begin{array}{c}\text { ID } \\
\begin{array}{c}\text { Feinstein } \\
\text { Kilambi }\end{array}\end{array}$ & $\begin{array}{l}\text { Other } \\
\text { designation }\end{array}$ & $\begin{array}{l}\text { Spectral } \\
\text { types }\end{array}$ & References \\
\hline 01 & HD 143 448 & B3e, B3IV, B3IVe, B2/B3III, B3, B1.5V, B1Ve & $1,2,3,4,5,6,10$ \\
02 & HD 143449 & B, B5IV, B5IV & $4,6,10$ \\
03 & HD 143 413 & B7IV & 4,10 \\
04 & CD-606021 & B8V & 10 \\
05 & CD-606007 & B9V & 10 \\
06 & HD 143 288 & B6Ve & 10 \\
07 & HD 143 340 & B9V, B5IV & 4,10 \\
08 & CD-606015 & B6V & 10 \\
11 & HD 143 287 & B8/B9Ib/II, B9IVp & 4,10 \\
12 & HD 143 309 & B8/B9Ib/II, B6III, B6V & $4,7,10$ \\
13 & CD-606011 & B8V & 10 \\
14 & CD-606013 & B8.5V & 10 \\
15 & CD-606017 & F0V & 10 \\
16 & CD-606027 & B8V & 10 \\
22 & HD 143 388 & B5V & 10 \\
23 & CD-606024 & A0V & 10 \\
24 & CPD-596562 & B9V & 10 \\
25 & HD 143 412 & B8, B8IIIp & 4,10 \\
26 & V 396 Nor & A0IV/V, A1V+A1V, A0V+A0V & $4,8,10$ \\
43 & HD 143 123 & B6II/III, B5V & 4,10 \\
86 & CPD-606287 & $\ldots$ & \\
90 & HD 143 027 & B8/9Ib/IIp, B8 (Si ) & 4,9 \\
\hline
\end{tabular}

Notes. Nomenclature according to Feinstein (1971) and Kilambi (1975).

References. (1) Merrill \& Burwell (1933); (2) Morris (1961); (3) Buscombe (1969); (4) Houk \& Cowley (1975); (5) Henize (1976); (6) Garrison et al. (1977); (7) Waelkens \& Rufener (1985); (8) González \& Levato (2006); (9) Renson \& Manfroid (2009); and (10) Grosso \& Levato (2011).
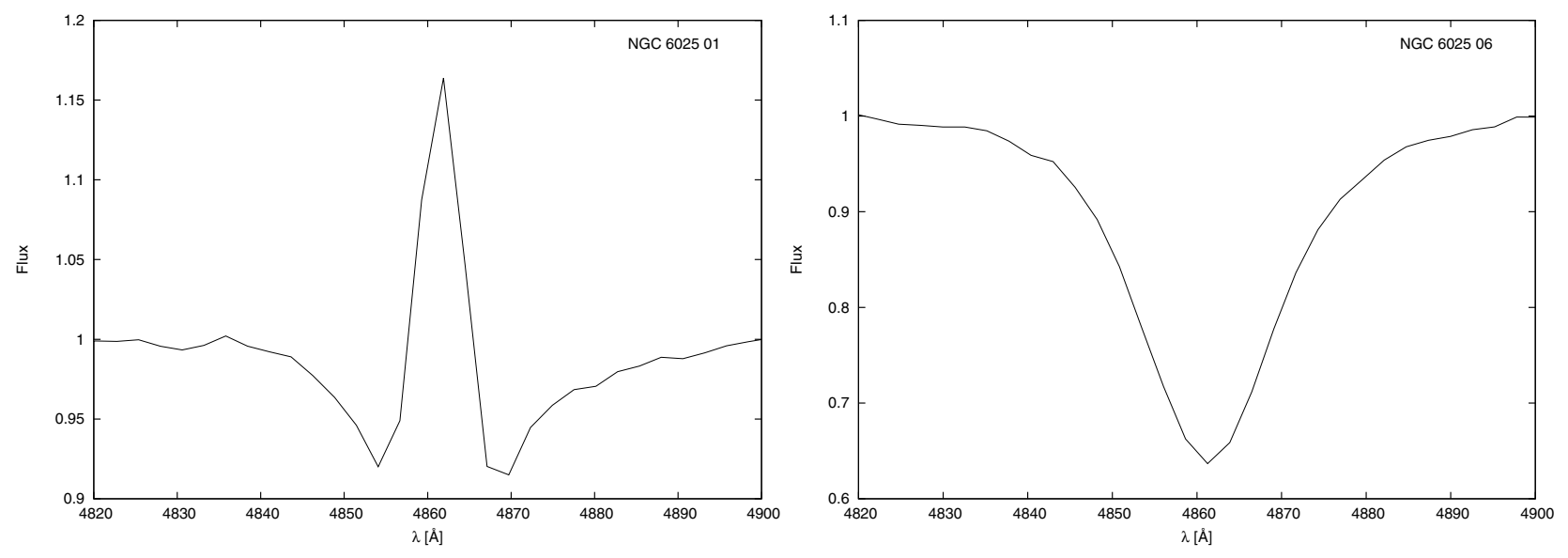

Fig. 2. $\mathrm{H} \beta$ profile of stars No. 1 (left) and No. 6 (right).

Pišmiš (1959) reported it as an open cluster for the first time. This cluster, together with Markarjan 18 and Pismis 16, seem to trace a defined filament that juts out from the local arm at $l=270^{\circ}$ and $d=1668 \mathrm{pc}$ (Moffat \& Vogt 1973a). On the other hand, according to Kopchev et al. (2006), Pismis 6 and Pismis 8 could be of similar ages, therefore, they might have been formed in the same giant molecular cloud and are good candidates for a binary system.

The distance of this cluster was obtained by different authors and various techniques. All the values agree very well (between 1.65 and $1.8 \mathrm{kpc})$. The colour excess $E(B-V)$ ranges from $0.28 \mathrm{mag}$ to $0.46 \mathrm{mag}$ and the cluster age is estimated between 1 and 38 Myr (Vogt \& Moffat 1973; Fitzgerald et al. 1979a; Forbes \& Short 1994; Rastorguev et al. 1999; Kharchenko et al. 2005, 2009b; Kopchev et al. 2006; Bukowiecki et al. 2011; Santos-Silva \& Gregorio-Hetem 2012).
There are only two spectroscopic studies of the cluster by Fitzgerald et al. (1979a,b). The first classifies stars Nos. 1, 2, 3 , and 4 as B2IV, B2IV, B1.5IV, and B1Vne, respectively. The second gives spectral types for the following stars: Nos. 1, 2, and 3 as B0.5V; No. 4 as B2IVe; stars Nos. 5 and 7 as B2V; star No. 6 as B4V, and star No. 51 as B9V. In the literature we find that stars Nos. 1 and 3 are classified as B1V (Garrison et al. 1977; Feast et al. 1961, respectively) but in the catalogue by Houk (1978), star No. 3 is listed as B3II.

HD 74180 is reported as a variable star in radial velocity (Moore et al. 1914), as a possible spectroscopic binary (Lunt 1918), as a visual binary (it has only one B2V-type companion, SLS 1114, separated by 37.5, Moffat \& Fitzgerald 1977), and as a $\alpha$ Cygni variable (van Leeuwen et al. 1998). This object is classified as: F2Ia (Bidelman 1951), F8 (Iriarte 1965), F3Ia 
Table 5. Collinder 223: Stellar fundamental parameters from the BCD system.

\begin{tabular}{|c|c|c|c|c|c|c|c|c|c|}
\hline $\begin{array}{r}\text { ID } \\
\text { Clar }\end{array}$ & des & $\begin{array}{c}D \\
{[\mathrm{dex}]}\end{array}$ & & $\begin{array}{ll}\Phi_{\mathrm{b}} & \text { S.T } \\
{[\mu]} & \end{array}$ & $\begin{array}{l}T_{\text {eff }} \\
{[\mathrm{K}]}\end{array}$ & $\begin{array}{l}\log g \\
\text { [dex] }\end{array}$ & $\begin{array}{c}M_{\mathrm{v}} \\
{[\mathrm{mag}]}\end{array}$ & $\begin{array}{c}M_{\mathrm{bol}} \\
{[\mathrm{mag}]}\end{array}$ & $\begin{array}{l}\Phi_{\mathrm{b}}^{0} \\
{[\mu]}\end{array}$ \\
\hline 002 & dd & 39 & 59 & $1.15 \mathrm{~B} 9$ & $844 \pm 299$ & $20 \pm 0.16$ & $0.25 \pm 0.25$ & $=0.23$ & $37 \pm 0$ \\
\hline 003 & TYC & 36 & 79 & 3 B9VI: & 4 & $9 \pm 0.07^{a}$ & $.20 \pm$ & $4^{a}$ & 13 \\
\hline 004 & HD 9 & 37 & 96 & $1.01 \mathrm{~B}^{2} \mathrm{~V}^{m k}$ & $13933 \pm 1$ & $4.04 \pm 0.00^{a}$ & $-0.88 \pm 0.32^{a}$ & -2.12 & $0.84 \pm$ \\
\hline 005 & TYC & 21 & 7 & $1.11 \mathrm{~B} 3 \mathrm{~V}$ & 5 & 8 & 3 & -2 & 0.74 \\
\hline 009 & $\mathrm{HD}$ ? & 2 & 61 & 1.26 A1IV & 9 & 4 & 1 & 0.86 & 1.09 \\
\hline 020 & HD 30 & 36 & 57 & $1.21 \mathrm{~B} 8 \mathrm{~V}$ & $12216 \pm$ & $4.18 \pm$ & $0.04=$ & -0.76 & 0.84 \\
\hline 021 & TYC 8613-1 & 0.47 & 74 & $1.21 \mathrm{~A} 1 \mathrm{~V}$ & 37 & $4.50 \pm 0.10:$ & $0.10 \pm$ & 0.43 & 1.04 \\
\hline 029 & HD 3 & 0.50 & 71 & $1.34 \mathrm{~A} 2: \mathrm{V}$ & 35: & $4.35 \pm 0.10:$ & $1.30 \pm$ & $1.10=$ & 1.09 \\
\hline 035 & $\mathrm{HD}$ ? & 16 & 6 & $1.36 \mathrm{~B} 3 \mathrm{II}$ & 82 & $2 \pm$ & $-4.07 \pm$ & -5.22 & $0.72=$ \\
\hline 048 & HD & 45 & 5 & $1.24 \mathrm{~A} 0 \mathrm{IV}$ & $10576 \pm$ & $4.02 \pm$ & 0. & -0.38 & $0.94=$ \\
\hline 049 & TYC & 41 & 54 & 1.20 B9IV & 30 & $3 \pm$ & 0 & -0.67 & 0.89 \\
\hline 056 & HD & 5 & 57 & $1.05 \mathrm{~B} 7 \mathrm{~V}$ & $66=$ & $4.15 \pm 0.16$ & $-0.07 \pm$ & -0.88 & $0.83=$ \\
\hline 057 & HD 3 & 42 & 69 & 1.18 A0V: & $11000 \pm$ & $4.40 \pm 0.11:$ & $0.36 \pm$ & $0.00 \pm$ & $0.93 \pm 0.04$ \\
\hline 058 & TY & .49 & 83 & 1.28 & $00 \pm 334^{a}$ & $4.20 \pm 0.03^{a}$ & $1.30 \pm 0.27^{a}$ & $1.10 \pm$ & $1.20 \pm 0.02^{m}$ \\
\hline 080 & HD $305218^{d d}$ & 0.08 & 82 & $1.24 \mathrm{~B} 0 \mathrm{~V}:{ }^{B e}$ & $30000 \pm 2000$ & $3.94 \pm 0.00^{a}$ & $-4.00 \pm 0.64^{a}$ & $-7.16 \pm 0.64^{a}$ & $0.57 \pm 0.02^{m}$ \\
\hline 105 & TYC & 0.29 & 54 & $1.32 \mathrm{~B} \mathrm{~V}^{p v}$ & $14650 \pm 617$ & $4.10 \pm 0.18$ & $-0.55 \pm 0.27$ & $-1.69 \pm 0.33$ & $0.78 \pm 0.01$ \\
\hline 106 & HD $305217^{d d}$ & 0.26 & 49 & 1.18 B5IV & $15891 \pm 593$ & $3.85 \pm 0.26$ & $-1.12 \pm 0.42$ & $-2.31 \pm 0.43$ & $0.77 \pm 0.01$ \\
\hline$\cdots$ & HD 3 & 0.17 & 39 & $1.41 \mathrm{~B} 3 \mathrm{III}$ & $18958 \pm 1616$ & $2.99 \pm 0.26$ & $-3.30 \pm 0.72$ & $-4.62 \pm 0.57$ & $0.73 \pm 0.01$ \\
\hline$\cdots$ & HD $305296^{d d}$ & 0.05 & 80 & $1.18{\mathrm{~B} 0 \mathrm{~V}^{B e}}^{3}$ & $30000 \pm 4275^{a}$ & $3.94 \pm 0.00^{a}$ & $-4.00 \pm 0.64^{a}$ & $-7.16 \pm 0.64^{a}$ & $0.57 \pm 0.02^{m}$ \\
\hline
\end{tabular}

Notes. Nomenclature according to Claria \& Lapasset (1991). The symbol : is used to indicate extrapolated values. $g$ is the stellar surface gravity given in $\mathrm{cm} \mathrm{s}^{-2}$. ${ }^{(d d)}$ Stars with double Balmer discontinuity. ${ }^{\left({ }^{m k}\right)}$ Spectral types derived using the MK-system. ${ }^{(B e)}$ Known Be star. ${ }^{\left({ }^{(p)}\right)}$ Identified as a photometric variable by Lapasset et al. (1991). ${ }^{(a)}$ Values interpolated from Cox (2000). ${ }^{(m)}$ Values determined using the relation between $\Phi_{\mathrm{b}}$ and $(B-V)$ given by Moujtahid et al. (1998).

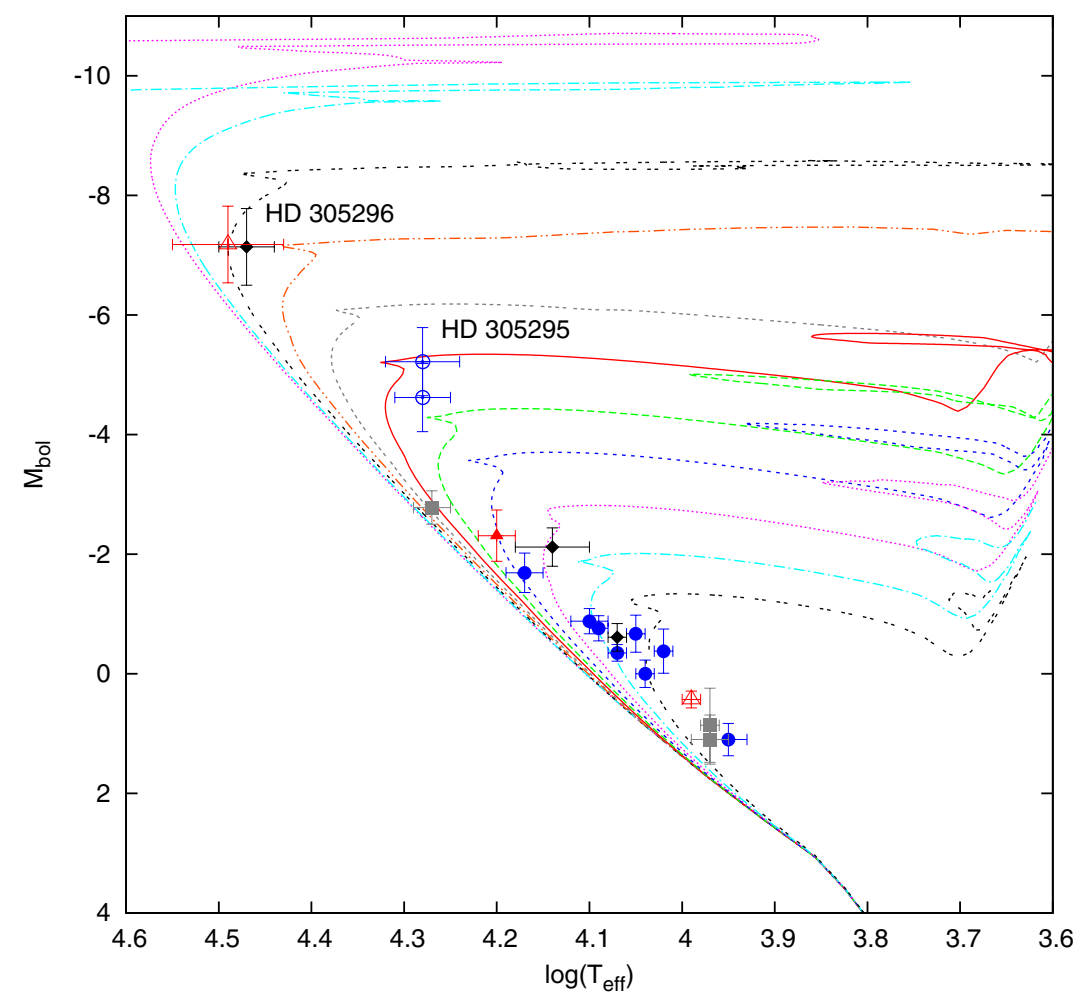

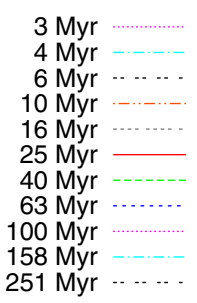

Fig. 3. Collinder 223: HR diagram. The age of this cluster is quite uncertain, from $25 \mathrm{Myr}$ to $136 \mathrm{Myr}$, since the brightest stars are pnm. The isochrone curves are given by Ekström et al. (2012). Probable members of the clusters are denoted in - (blue) symbols, pnm in $\odot$ (blue) and non-members in (grey). Be star cluster members are indicated in $\Delta$ (red), Be pnm in $\Delta$ (red), and Be non-members in (black).
(Malaroda 1975), F0Ia (Humphreys 1978), and F4I (Mersch \& Heck 1980).

We report BCD parameters of stars of our sample and their associated quantities (see Tables 9 and 10). From the second table, we derive the mean parameters of this cluster (including members and pnm $), \overline{\left(m_{\mathrm{v}}-M_{\mathrm{v}}\right)_{0}}=12.18 \pm 0.30 \mathrm{mag}$, which corresponds to a distance of $2726 \mathrm{pc}, \overline{E(B-V)}=0.58 \pm 0.05$ and $\bar{d}=2920 \pm 340 \mathrm{pc}$.

We recognize a population of early B-type stars. From the HR diagram (see Fig. 5), using the method of isochrones and considering the position of the more evolved stars, we estimate an age between 6 and 10 Myr. On the other hand, 

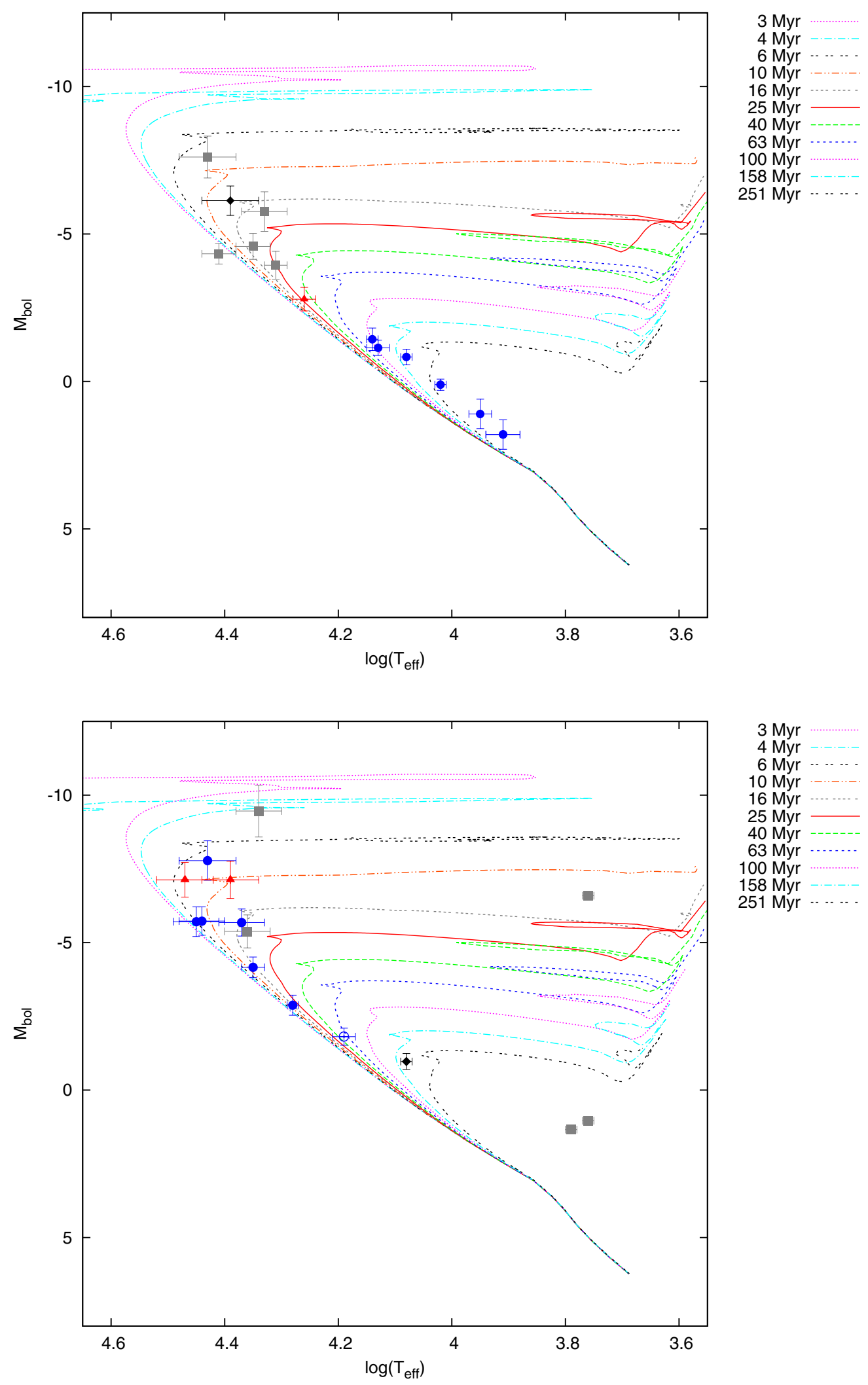

Fig. 4. Hogg 16: HR diagram. We recognize two stellar populations: Hogg 16a and Hogg 16b represented in - (blue) and - (grey) symbols, respectively. Be star cluster members are indicated in $\Delta$ (red) for Hogg 16a and in (black) for Hogg 16b. The isochrone curves are taken from Ekström et al. (2012).

Fig. 5. NGC 2645: HR diagram. The estimated age of this cluster is around 6-10 Myr. The isochrone curves are taken from Ekström et al. (2012). Probable members of the clusters are denoted in filled $\bullet$ (blue) symbols, pnm in $\odot$ (blue), and nonmembers in (grey). Be star cluster members are indicated in $\boldsymbol{\Delta}$ (red) and Be non-members in (black).

taking the average age of individual members (see Table 10) we calculate $77 \pm 60$ Myr.

Our age determinations are in very good agreement with values found in the literature (Forbes \& Short 1994; Kopchev et al. 2006; Bukowiecki et al. 2011; Santos-Silva $\&$ Gregorio-Hetem 2012). However, we find a true distance modulus greater than 0.5 mag compared with previous determinations (11.2-11.57 mag) locating the cluster far away and in a higher reddening region.

Regarding the star membership of the cluster, the BCD distance-moduli point out that stars Nos. 1, 51, 56, 72, and HD 74180 are non-members, while No. 10 is a pnm. Star No. 56 
Table 6. Collinder 223: Physical parameters and distance to stars.

\begin{tabular}{|c|c|c|c|c|c|c|c|c|}
\hline $\begin{array}{c}\text { ID } \\
\text { Claria } \\
\end{array}$ & $\begin{array}{l}\text { Other } \\
\text { designation }\end{array}$ & $\begin{array}{c}m_{\mathrm{v}} \\
{[\mathrm{mag}]}\end{array}$ & V) & $\begin{array}{c}\left(m_{\mathrm{v}}-M_{\mathrm{v}}\right)_{0} \\
{[\mathrm{mag}]}\end{array}$ & $\begin{array}{c}d \\
{[\mathrm{pc}]}\end{array}$ & $\begin{array}{l}\log \mathcal{L} \\
{\left[\mathcal{L}_{\odot}\right]}\end{array}$ & $\begin{array}{c}M \\
{\left[M_{\odot}\right]}\end{array}$ & $\begin{array}{c}\text { Age } \\
{[\mathrm{Myr}]}\end{array}$ \\
\hline 002 & HD $91113^{d d}$ & 68 & .02 & $8.84 \pm 0.3^{n m}$ & $587 \pm 69$ & $2.14 \pm 0.09$ & $10 \pm 0.18$ & $137 \pm 64$ \\
\hline 003 & TY & & & & 209 & 18 & 3 & \\
\hline 004 & HD $91041^{d d}$ & 8.16 & $0.12 \pm 0.02$ & $8.67 \pm 0.3^{n m}$ & $542 \pm 80$ & $2.74 \pm 0.13$ & $4.26 \pm 0.35$ & $99 \pm 23$ \\
\hline 005 & TYC & 1.61 & .01 & 12.25 & 2825 & $3.01 \pm$ & $5.78=$ & $10=$ \\
\hline 009 & HD 3 & 11.24 & .05 & $.4^{\mathrm{pnm}}$ & $769 \pm 157$ & $1.55 \pm$ & $2.28 \pm$ & $370 \pm 118$ \\
\hline 020 & & 11.53 & $25 \pm$ & $10.72 \pm 0.2$ & $1390 \pm 128$ & $2.20 \pm 0.08$ & $3.24 \pm 0.18$ & $124 \pm 55$ \\
\hline 021 & $\mathrm{~T}$ & 12.44 & & $11.95 \pm$ & $2457 \pm$ & $1.72 \pm$ & $2.26=$ & $186 \pm 188$ \\
\hline 029 & & 10.42 & & & & $1.46 \pm$ & & $198 \pm 194$ \\
\hline 035 & & & & 12.5 & $261 \pm 1245$ & .23 & $13.90=$ & \\
\hline & & & & & & 15 & & \\
\hline 049 & & 4 & & & & $2.16=$ & $3.13=$ & 197 \\
\hline 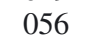 & & & & & & $2.25=$ & 3.3 & $11^{\prime}$ \\
\hline 7 & & & & & & $1.90=$ & 2.62 & 116 \\
\hline 058 & & 9 & & & 9 & 1.46 & $2.10=$ & 122 \\
\hline nen & $\mathrm{HL}$ & 7 & & 12.9 & 77 & $4.76=$ & 17.56 & \\
\hline 105 & TY & 6 & & & 1570 & $2.57=$ & $4.26=$ & 66 \\
\hline 106 & & & & & 4 & $2.82=$ & 5.20 & \\
\hline$\ldots$ & & 10 & & 12.70 & $3474 \pm 1$ & $3.74=$ & 11.74 & $22 \pm 11$ \\
\hline$\ldots$ & HD $305296^{d d}$ & 9.26 & $0.41 \pm 0.02$ & $11.99 \pm 0.6^{p n m}$ & $2499 \pm 747$ & $4.76 \pm 0.26$ & $17.77 \pm 3.28$ & $7 \pm 2$ \\
\hline
\end{tabular}

Notes. $m_{\mathrm{v}}$ Values obtained from SIMBAD database. ${ }^{(d d)}$ Stars with double Balmer discontinuity. ${ }^{(n m)}$ Non-member stars. ${ }^{(p n m)}$ Probable non-member stars.

Table 7. Hogg 16: Stellar fundamental parameters from the BCD system.

\begin{tabular}{|c|c|c|c|c|c|c|c|c|c|c|}
\hline $\begin{array}{c}\text { ID } \\
\text { Vázquez }\end{array}$ & $\begin{array}{l}\text { Other } \\
\text { designation }\end{array}$ & $\begin{array}{c}D \\
{[\mathrm{dex}]}\end{array}$ & $\begin{array}{c}\lambda_{1} \\
[\AA]]\end{array}$ & $\begin{array}{l}\Phi_{\mathrm{b}} \\
{[\mu]}\end{array}$ & S & $\begin{array}{l}T_{\text {eff }} \\
{[\mathrm{K}]}\end{array}$ & $\begin{array}{l}\log g \\
{[\operatorname{dex}]}\end{array}$ & $\begin{array}{c}M_{\mathrm{v}} \\
{[\mathrm{mag}]}\end{array}$ & $\begin{array}{c}M_{\mathrm{bol}} \\
{[\mathrm{mag}]}\end{array}$ & $\begin{array}{l}\Phi_{\mathrm{b}}^{0} \\
{[\mu]}\end{array}$ \\
\hline 01 & CD-604699 & 0.14 & 71 & 1.45 & B2VI: & $455 \pm 1586$ & $4.24 \pm 0.06:$ & $2.25 \pm 0.25:$ & $-4.33 \pm 0.35$ & $.70 \pm 0.01$ \\
\hline 02 & & 2 & 39 & 1.57 & B2II & $518 \pm 2177$ & $2.80 \pm 0.17:$ & $-4.51=$ & -5.76 & 0.70 \\
\hline 03 & $\mathrm{CD}-$ & 0.10 & 46 & 1.54 & $\mathrm{~B} 1 \mathrm{III}{ }^{B e}$ & 826 & $2.98 \pm 0.34$ & -4.38 & -6.13 & $0.69 \pm$ \\
\hline 05 & & 0.43 & 73 & 1.58 & & & $4.52 \pm$ & & & \\
\hline 06 & & 0.18 & 49 & 1.52 & & & 31 & & -3. & .01 \\
\hline 09 & & 0.07 & 39 & 2.19 & $\mathrm{~B} 0 \mathrm{Ib}^{E m}$ & & & & -7.61 & \\
\hline 14 & & 0.3 & 52 & 1.20 & & & & & & \\
\hline 49 & & & 87 & 1.30 & A & & & & & \\
\hline 50 & & & 53 & 1.22 & & & & & & \\
\hline$=0$ & & & 49 & & & & & & & \\
\hline 53 & & & 49 & 1.50 & B2 & & & & & \\
\hline 68 & & & 52 & 1.08 & B4 & & & & & \\
\hline 14 & & & 73 & 1.5 & & & & & & \\
\hline 75 & CPD-604705 & .42 & 88 & 1.66 & A5V: & $3180 \pm 500^{a}$ & $4.29 \pm 0.30^{a}$ & $1.95 \pm 0.50^{a}$ & $1.80 \pm 0.50^{a}$ & $1.27 \pm 0.0$ \\
\hline
\end{tabular}

Notes. Nomenclature according to Vazquez \& Feinstein (1991). The symbol : is used to indicate extrapolated values. $g$ is the stellar surface gravity given in $\mathrm{cm} \mathrm{s}^{-2}$. ${ }^{(d d)}$ Stars with double Balmer discontinuity. ${ }^{(m k)}$ Spectral types derived using the MK-system. ${ }^{(B e)}$ Known Be star. ${ }^{(E m)}$ Emission line star. ${ }^{(a)}$ Values interpolated from Cox (2000).

is considered a non-member because we classify it as F7III and it has a similar apparent visual magnitude as a B3V star member. We agree with Fitzgerald et al. (1979a) that star No. 51 is outside the cluster and also confirm that HD 74180 is a non-member.

The brighter cluster population consists of giant and supergiant B early-type stars. We have determined the spectral type of five stars for the first time. For stars Nos. 5, 6, and 51 our classification agrees very well with that done by Fitzgerald et al. (1979b), but even though the spectral types of stars Nos. 1, 2, 3, 4 , and 7 also show good agreement, we find that those are more evolved. We also confirm the supergiant nature of HD 74180 and determine an F8Ib spectral type. Particularly, for this star, Steemers \& van Genderen (1986) found variations in brightness (by $\sim 0.06$ in $V$ ) and colours (by $\sim 0.02$ ), which were confirmed by HIPPARCos data (van Leeuwen et al. 1998), we believe that these variations could explain the observed discrepancies in the spectral type.

In addition to the known Be stars Nos. 3 and 4, in our sample we distinguish two other stars showing the second Balmer discontinuity: No. 51 and the known supergiant HD 74 180. The former is a field Be candidate of spectral type B8IV.

\subsection{NGC 3114}

NGC $3114\left(\alpha=10^{\mathrm{h}} 02^{\mathrm{m}} 36^{\mathrm{s}}\right.$ and $\left.\delta=-60^{\circ} 07^{\mathrm{m}} 2 ; 2000\right)$ is located in a crowded low-reddened field projected on to the Carina complex, which complicates the separation of cluster members from the field stars. The cluster is reported to be rich in peculiar 
Table 8. Hogg 16: Physical parameters and distance to stars.

\begin{tabular}{clccccccc}
\hline \hline $\begin{array}{c}\text { ID } \\
\text { Vázquez }\end{array}$ & $\begin{array}{l}\text { Other } \\
\text { designation }\end{array}$ & $\begin{array}{c}m_{\mathrm{v}} \\
{[\mathrm{mag}]}\end{array}$ & $E(B-V)$ & $\begin{array}{c}\left(m_{\mathrm{v}}-M_{\mathrm{v}}\right)_{0} \\
{[\mathrm{mag}]}\end{array}$ & $\begin{array}{c}d \\
{[\mathrm{pc}]}\end{array}$ & $\begin{array}{c}\log \mathcal{L} \\
{\left[\mathcal{L}_{\odot}\right]}\end{array}$ & $\begin{array}{c}M \\
{\left[M_{\odot}\right]}\end{array}$ & $\begin{array}{c}\text { Age } \\
{[\mathrm{Myr}]}\end{array}$ \\
\hline 01 & CD-604699 & 10.43 & $0.51 \pm 0.01$ & $11.11 \pm 0.3$ & $1669 \pm 195$ & $3.63 \pm 0.14$ & $9.36 \pm 1.11$ & $2 \pm 3$ \\
02 & CD-604696 & 10.48 & $0.59 \pm 0.01$ & $13.16 \pm 0.8$ & $4279 \pm 1691$ & $4.20 \pm 0.27$ & $20.06 \pm 11.92$ & $12 \pm 7$ \\
03 & CD-604701dd & 10.17 & $0.57 \pm 0.01$ & $12.77 \pm 0.8$ & $3577 \pm 1304$ & $4.35 \pm 0.20$ & $28.10 \pm 28.90$ & $9 \pm 5$ \\
05 & CPD-604698 & 11.06 & $0.40 \pm 0.03$ & $9.88 \pm 0.4$ & $945 \pm 180$ & $1.85 \pm 0.08$ & $2.44 \pm 0.28$ & $140 \pm 141$ \\
06 & CPD-604695 & 11.36 & $0.54 \pm 0.01$ & $12.01 \pm 0.5$ & $2526 \pm 599$ & $3.47 \pm 0.19$ & $8.10 \pm 1.06$ & $22 \pm 6$ \\
09 & CPD-604703 & 10.85 & $1.03 \pm 0.01$ & $13.79 \pm 0.9$ & $5715 \pm 2480$ & $4.94 \pm 0.28$ & $\cdots$ & $5 \pm 3$ \\
14 & CPD-604699 & 10.28 & $0.22 \pm 0.01$ & $9.64 \pm 0.3$ & $846 \pm 103$ & $2.23 \pm 0.10$ & $3.32 \pm 0.18$ & $155 \pm 33$ \\
49 & HD 117239 & 10.22 & $0.12 \pm 0.02$ & $8.55 \pm 0.5$ & $512 \pm 119$ & $1.46 \pm 0.20$ & $2.12 \pm 0.19$ & $387 \pm 226$ \\
50 & HD 117256 & 10.18 & $0.29 \pm 0.01$ & $9.63 \pm 0.3$ & $842 \pm 102$ & $2.35 \pm 0.10$ & $3.85 \pm 0.27$ & $91 \pm 25$ \\
52 & HD 116999 & 9.49 & $0.35 \pm 0.01$ & $9.04 \pm 0.4$ & $642 \pm 106$ & $2.47 \pm 0.15$ & $4.20 \pm 0.38$ & $92 \pm 14$ \\
53 & CPD-604719 & 11.00 & $0.54 \pm 0.01$ & $12.20 \pm 0.5$ & $2749 \pm 696$ & $3.73 \pm 0.18$ & $10.83 \pm 2.81$ & $15 \pm 5$ \\
68 & HD 116875dd & 7.30 & $0.23 \pm 0.01$ & $8.02 \pm 0.4$ & $402 \pm 73$ & $3.01 \pm 0.16$ & $6.04 \pm 0.58$ & $32 \pm 11$ \\
74 & CPD-604711 & 10.73 & $0.21 \pm 0.04$ & $8.13 \pm 0.5$ & $422 \pm 98$ & $1.18 \pm 0.20$ & $1.82 \pm 0.15$ & $484 \pm 369$ \\
75 & CPD-604705 & 11.16 & $0.27 \pm 0.04$ & $8.37 \pm 0.5$ & $473 \pm 110$ & $1.18 \pm 0.20$ & $1.82 \pm 0.15$ & $484 \pm 369$ \\
\hline
\end{tabular}

Notes. $m_{\mathrm{v}}$ Values obtained from SIMBAD database. ${ }^{(d d)}$ Stars with double Balmer discontinuity.

Table 9. NGC 2645: Stellar fundamental parameters from the BCD system.

\begin{tabular}{|c|c|c|c|c|c|c|c|c|c|c|}
\hline $\begin{array}{c}\text { ID } \\
\text { Forbes }\end{array}$ & $\begin{array}{l}\text { Other } \\
\text { designation }\end{array}$ & $\begin{array}{c}D \\
{[\mathrm{dex}]}\end{array}$ & $\begin{array}{l}\lambda_{1} \\
{[\AA]}\end{array}$ & $\begin{array}{l}\Phi_{\mathrm{b}} \\
{[\mu]}\end{array}$ & S.T & $\begin{array}{l}T_{\text {eff }} \\
{[\mathrm{K}]}\end{array}$ & $\begin{array}{l}\log g \\
{[\mathrm{dex}]}\end{array}$ & $\begin{array}{c}M_{\mathrm{v}} \\
{[\mathrm{mag}]}\end{array}$ & $\begin{array}{c}M_{\mathrm{bol}} \\
{[\mathrm{mag}]}\end{array}$ & $\begin{array}{l}\Phi_{\mathrm{b}}^{0} \\
{[\mu]}\end{array}$ \\
\hline 01 & HD 73919 & 0.05 & 28 & 1.30 & B1Ia & $22067 \pm 2210$ & $2.74 \pm 0.10$ & $-7.48 \pm 0.67:$ & $-9.46 \pm 0.88$ & $0.70 \pm 0.01$ \\
\hline 02 & $\mathrm{CD}-4$ & 0.05 & 40 & 1.61 & BOIb & $6920 \pm 3565$ & $2.67 \pm 0.12:$ & $-6.20 \pm 0.72:$ & $-7.78 \pm 0.67$ & $0.68 \pm 0.01$ \\
\hline 03 & HD 73903 & 0.07 & 42 & 1.65 & $\mathrm{~B} 0 \mathrm{II}^{B e}$ & $4750 \pm 3255$ & $2.78 \pm 0.19$ & $-5.25 \pm 0.96$ & $-7.13 \pm 0.63$ & $0.68 \pm 0.01$ \\
\hline 04 & CD-45 4355 & 0.07 & 48 & 1.27 & $\mathrm{~B} 0 \mathrm{II}^{B e}$ & $9375 \pm 4023$ & $2.94 \pm$ & $-4.88 \pm 0.84$ & $-7.13 \pm 0.59$ & $0.68 \pm 0.01$ \\
\hline 05 & $\mathrm{CPD}-$ & 0.10 & 55 & 1.32 & B1IV & $28188 \pm 2425$ & $3.83 \pm$ & $-3.36 \pm$ & $-5.71 \pm$ & $0.69 \pm 0.01$ \\
\hline 06 & $\mathrm{CD}-$ & 0.21 & 60 & 1.69 & B3V & $18953 \pm 647$ & $4.16 \pm$ & -1.50 & $-2.88 \pm$ & $0.73 \pm 0.01$ \\
\hline 07 & CPD & 0.10 & 51 & 1.84 & B1III & $8 \pm 2678$ & $3.43 \pm$ & $-3.53=$ & -5.73 & $0.69 \pm 0.01$ \\
\hline 10 & $\mathrm{CD}-$ & 0.28 & 55 & 1.98 & B5V & $15372 \pm 624$ & $4.10 \pm$ & $-0.68 \pm 0.28$ & $-1.81 \pm$ & $0.77 \pm 0.01$ \\
\hline 51 & $\mathrm{CPD}$ & 0.38 & 50 & 1.62 & B8IV & $12134 \pm$ & $3.91 \pm$ & $-0.22=$ & $-0.97=$ & $0.85 \pm 0.01$ \\
\hline 55 & $51-2888-1$ & 0.12 & 44 & 0.90 & B2III & $22672 \pm 2095$ & $3.02 \pm 0.32$ & $-3.70 \pm 0.71$ & $-5.38 \pm 0.56$ & $0.70 \pm 0.01$ \\
\hline 56 & $51-474-1$ & 0.20 & 44 & 1.94 & F7III & $5717 \pm 83:^{a}$ & $3.01 \pm 0.02^{a}$ & $1.17 \pm 0.03:^{a}$ & $1.04 \pm 0.03: a$ & $2.31 \pm 0.01$ \\
\hline 58 & ALS 1 & 0.11 & 46 & 1.30 & B1III & $23393 \pm 2165$ & $3.11 \pm 0.35$ & $-3.74 \pm 0.72$ & $-5.68 \pm 0.46$ & $0.70 \pm 0.01$ \\
\hline 59 & J0839 & 0.16 & 54 & 1.41 & B2IV & $22145 \pm 1138$ & $3.93 \pm$ & $-2.33 \pm 0.43$ & $-4.16 \pm 0.35$ & $0.71 \pm 0.00$ \\
\hline 72 & $\mathrm{CD}-$ & 0.30 & 61 & 2.03 & F2IV & $6133 \pm 83:^{a}$ & $3.12 \pm 0.02^{a}$ & $1.33 \pm 0.03:^{a}$ & $1.34 \pm 0.03:^{a}$ & $1.86 \pm 0.07$ \\
\hline & HD $74180^{d d}$ & 0.24 & 22 & 2.67 & F8Ib & $5750 \pm 198^{a}$ & $1.38 \pm 0.02^{a}$ & $-6.50 \pm 0.04^{a}$ & $-6.59 \pm 0.04^{a}$ & $2.51 \pm 0.14$ \\
\hline
\end{tabular}

Notes. Nomenclature according to Forbes \& Short (1994). The symbol : is used to indicate extrapolated values. $g$ is the stellar surface gravity given in $\mathrm{cm} \mathrm{s}^{-2}$. ${ }^{(d d)}$ Stars with double Balmer discontinuity. ${ }^{(B e)}$ Known Be stars. ${ }^{(a)}$ Values interpolated from Cox (2000).

stars like Ap, Bp, and Be (Levato \& Malaroda 1975). According to Carraro \& Patat (2001), it is possible to separate the cluster into two populations: several low-reddened stars that are presumably cluster members and field stars with larger reddening.

The parameters of this cluster were derived using different techniques. Photometric studies were carried out by Lyngå (1962), Jankowitz \& McCosh (1962), Becker \& Fenkart (1971), Schmidt (1982), Schneider \& Weiss (1988), Sagar \& Sharples (1991), Malysheva (1997), Tadross (2001) and Carraro \& Patat (2001). Several works derived the cluster parameters from HIPPARCos mission (Robichon et al. 1999; Rastorguev et al. 1999; Loktin \& Beshenov 2001; Kharchenko et al. 2005, 2009a) or used either spectroscopic methods or combinations of two or more techniques (Amieux \& Burnage 1981; Lindoff 1968; González \& Lapasset 2001).

Distance modulus determinations range from $9.45 \mathrm{mag}$ to $10.26 \mathrm{mag}$, which correspond to distances between $840 \mathrm{pc}$ and $1130 \mathrm{pc}$. The cluster age has large uncertainties with values between $51 \mathrm{Myr}$ and 500 Myr. Regarding the colour excess, all authors obtain values between 0 and 0.08 mag.
The spectroscopic studies, mainly related to stellar classifications, were done by Frye et al. (1970), Levato \& Malaroda (1975) and González \& Lapasset (2001). Other spectral classifications of individual stars were also found in the literature (Houk \& Cowley 1975; Amieux \& Burnage 1981; Jaschek \& Egret 1982; Mermilliod 1982a; Gray \& Garrison 1989; Hamdy et al. 1993; Nesterov et al. 1995; Renson \& Manfroid 2009; Bychkov et al. 2009). All these data are shown in Table 2.

The results from the BCD method are listed in Table 11 and the derived values are in Table 12. The probable members are selected from Fig. 1. There we clearly identify a high concentration of stars around the distance modulus $\sim 9$ mag. The mean cluster parameters determined (taking into account members and pnm) are: $\overline{E(B-V)}=0.10 \pm 0.01 \mathrm{mag}, \overline{\left(m_{\mathrm{v}}-M_{\mathrm{v}}\right)_{0}}=$ $9.20 \pm 0.15 \mathrm{mag}$, which corresponds to $\langle d\rangle=694 \mathrm{pc}$, and a mean distance $\bar{d}=704 \pm 103 \mathrm{pc}$. According to our criterion, we find that stars Nos. 4, 33, 66, 105, and 132 are pnm, while Nos. 9, 100,102 , and 141 are non-member stars.

It is interesting to stress that stars of this cluster are characterized by an anomalous colour gradient (see Table 3 and Sect. 4). 
Table 10. NGC 2645: Physical parameters and distances to stars.

\begin{tabular}{|c|c|c|c|c|c|c|c|c|}
\hline $\begin{array}{c}\text { ID } \\
\text { Forbes }\end{array}$ & $\begin{array}{l}\text { Other } \\
\text { designation }\end{array}$ & $\begin{array}{c}m_{\mathrm{v}} \\
{[\mathrm{mag}]}\end{array}$ & $E(B-V)$ & $\begin{array}{c}\left(m_{\mathrm{v}}-M_{\mathrm{v}}\right)_{0} \\
{[\mathrm{mag}]}\end{array}$ & $\begin{array}{c}d \\
{[\mathrm{pc}]}\end{array}$ & $\begin{array}{l}\log \mathcal{L} \\
{\left[\mathcal{L}_{\odot}\right]}\end{array}$ & $\begin{array}{c}M \\
{\left[M_{\odot}\right]}\end{array}$ & $\begin{array}{c}\text { Age } \\
{[\mathrm{Myr}]}\end{array}$ \\
\hline 01 & HD 73919 & 8.85 & $0.41 \pm 0.01$ & $15.06 \pm 0.7^{n m}$ & $10275 \pm 3174$ & $5.68 \pm 0.35$ & $35.28 \pm 9.72$ & $5 \pm 1$ \\
\hline 02 & CD-45 4352 & .91 & 0.63 & $13.16 \pm 0.7$ & $4280 \pm 1420$ & $5.01 \pm 0.27$ & $\cdots$ & \\
\hline 03 & HD 73903 & 9.05 & $0.65 \pm 0.01$ & $12.24 \pm 0.96$ & $2799 \pm 1238$ & $4.75 \pm 0.25$ & $2.73 \pm 43$ & $=4$ \\
\hline 04 & CD-45 4355 & 9.47 & $0.40 \pm 0.01$ & $13.11 \pm 0.8$ & $4188 \pm 1621$ & $4.75 \pm 0.24$ & $\cdots$ & $5 \pm 3$ \\
\hline 05 & CPD-45 2779 & 10.62 & $0.43 \pm 0.01$ & $12.65 \pm 0.5$ & $3384 \pm 843$ & $4.18 \pm 0.20$ & $14.64 \pm 3.32$ & $6 \pm 3$ \\
\hline 06 & $\mathrm{CD}-454351 \mathrm{E}$ & 11.50 & $0.65 \pm 0.01$ & $10.99 \pm 0.2$ & $1574 \pm 175$ & $3.05 \pm 0.14$ & $6.07 \pm 0.38$ & $17 \pm 11$ \\
\hline 07 & CPD-45 2774 & 11.14 & $0.78 \pm 0.01$ & $12.25 \pm 0.6$ & $2821 \pm 819$ & $4.19 \pm 0.19$ & $18.78 \pm 10.44$ & $7 \pm 4$ \\
\hline 10 & $\mathrm{CD}-454351 \mathrm{~J}$ & 12.30 & $0.82 \pm 0.01$ & $10.44 \pm 0.3^{p n m}$ & $1223 \pm 159$ & $2.62 \pm 0.12$ & $4.52 \pm 0.26$ & $52 \pm 21$ \\
\hline 51 & CPD-45 2775 ${ }^{d d}$ & 11.20 & $0.53 \pm 0.01$ & $9.78 \pm 0.3^{n m}$ & $902 \pm 138$ & $2.28 \pm 0.11$ & $3.52 \pm 0.25$ & $159 \pm 15$ \\
\hline 55 & TYC 8151-2888-1 & 10.97 & $0.13 \pm 0.01$ & $14.27 \pm 0.7$ & $7135 \pm 2335$ & $4.05 \pm 0.22$ & $17.61 \pm 11.12$ & $13 \pm 6$ \\
\hline 56 & TYC 8151-474-1 & 11.30 & $-0.25 \pm 0.01$ & $\ldots n m$ & $\cdots$ & $1.48 \pm 0.01$ & $2.06 \pm 0.16$ & $640 \pm 170$ \\
\hline 58 & ALS 17179 & 9.94 & $0.41 \pm 0.01$ & $12.41 \pm 0.7$ & $3032 \pm 1006$ & $4.17 \pm 0.18$ & $17.71 \pm 10.87$ & $12 \pm 5$ \\
\hline 59 & J06393443-4618457 & 11.51 & $0.48 \pm 0.01$ & $12.35 \pm 0.4$ & $2954 \pm 586$ & $3.56 \pm 0.14$ & $8.68 \pm 0.96$ & $16 \pm 6$ \\
\hline 72 & CD-45 4366 & 10.23 & $0.12 \pm 0.01$ & $8.53 \pm 0.04^{n m}$ & $508 \pm 10$ & $1.36 \pm 0.01$ & $1.96 \pm 0.10$ & $860 \pm 350$ \\
\hline$\cdots$ & HD $74180^{d d}$ & 3.82 & $0.11 \pm 0.01$ & $9.98 \pm 0.05^{n m}$ & $990 \pm 23$ & $4.53 \pm 0.02$ & $12.63 \pm 1.04$ & \\
\hline
\end{tabular}

Notes. $m_{\mathrm{v}}$ Values obtained from SIMBAD database. ${ }^{(d d)}$ Stars with double Balmer discontinuity. ${ }^{(n m)}$ Non-member stars. ${ }^{(p n m)}$ Probable non-member stars.

We find that about $50 \%$ of the studied stars have a negative index $\left(\Phi_{\mathrm{b}}-\Phi_{\mathrm{b}}^{0}\right)$, therefore, we are not able to derive colour excesses and distances for the entire sample.

In Fig. 6 we plot the stars in an HR diagram together with the isochrones given by Ekström et al. (2012). Stars with later spectral types are shifted redwards from the main sequence, as if they were more evolved, so that it is very difficult to assign a unique age to the cluster as a whole. The spread of ages, between $6 \mathrm{Myr}$ and $500 \mathrm{Myr}$, are also found from the calculation of individual ages as is shown in Table 12.

The mean true distance modulus derived from the BCD method agrees well with the value determined by Loktin \& Beshenov (2001, 9.445 mag) using the HIPPARCos trigonometric parallaxes. However, we are not able to define the age since the region seems to have a mixture of stars with different ages. Several authors were faced with the same problem when using photometric data. Our colour excess estimate agrees quite well with the values obtained by Jankowitz \& McCosh (1962, $0.09 \mathrm{mag}$ ), Schmidt (1982, $0.08 \mathrm{mag}$ ), and Kharchenko et al. (2009a, $0.08 \mathrm{mag}$ ), although we were not able to use all the stars observed in NGC 3114.

We have classified four stars for the first time. For most of the stars, the derived spectral types agree with previous determinations (with discrepancies of at least two sub-spectral types). In our sample, there are three stars described as peculiar objects: Nos. 4, 5, and 9 (Levato \& Malaroda 1975; Amieux \& Burnage 1981; Schneider \& Weiss 1988). Stars Nos. 5 and 9 reported as blue stragglers by Schmidt (1982) and Ahumada \& Lapasset (1995), respectively, show colour gradient excesses equal zero or negative. Instead, the peculiarity observed in No. 4 is under debate. On the one hand, Levato \& Malaroda (1975) classified it as B8.5IV ( $\mathrm{Si}$ II) CP2 star, which was later confirmed by Amieux \& Burnage (1981). On the other hand, Schneider \& Weiss (1988) report that their photometric data do not match the colours expected for a CP star. In addition, Maitzen et al. (1988), based on the spectral classification done by Frye et al. (1970), argue that this star would not be peculiar because lines of Silicon are typically associated with the luminosity class III, which agrees with the luminosity class we derived.

In relation to membership of stars, it was early reported that stars Nos. 3, 5, 7, 79, 102, 132, HD 87 436, and HD 87801 are non-members (Frye et al. 1970; Schneider \& Weiss 1988; Maitzen et al. 1988; González \& Lapasset 2001) while stars Nos. 91, 105, and 130 are pnm (Schneider \& Weiss 1988). From our results, we confirm that the star No. 102 is a non-member, while stars Nos. 105, 132, and 147 are pnm. However, we find that Nos. 79, 91, and 130 are members. For the rest of the sample (Nos. 3, 5, 7, HD 87 436, and HD 87 801) we cannot confirm their memberships since we do not calculate their distances because they have negative colour gradients.

In addition to the known Be stars (Nos. 28, 33, 47, 129, and HD 87801 ), in our sample we distinguish nine more Be candidate stars, namely: Nos. 3, 4, 6, 11, 79, 91, 126, 132, and HD 87436, since they show a second Balmer discontinuity. Particularly, stars Nos. 3, 4, and 91 were reported as line emission stars by González \& Lapasset (2001).

The stars proposed as blue stragglers, Nos. 3, 5, 9, 10, 15, and 124 (Schmidt 1982; Ahumada \& Lapasset 1995), should be studied in more detail considering they were reported as non-members, show negative gradient excesses or are low-luminosity stars (see Table 11).

\subsection{NGC 6025}

NGC $6025\left(\alpha=16^{\mathrm{h}} 03^{\mathrm{m}} 17^{\mathrm{s}}\right.$ and $\delta=-60^{\circ} 25^{\mathrm{m}} 9$; 2000$)$ appears to have a well defined $M_{\mathrm{v}}$ vs. $(B-V)_{0}$ diagram. Instead, the $M_{\mathrm{v}}$ vs. $(U-B)_{0}$ plane shows a kind of splitting (or gap) of the main sequence at absolute magnitudes $M_{\mathrm{v}}<1$ (cf. Mermilliod 1981). This gap, which was also reported by Kilambi (1975) in the interval $0.1<(B-V)_{0}<0.2$, is not attributed to mixing of clusters at different ages (Mermilliod 1981).

Photometric studies of this cluster were carried out by Hogg (1953), Feinstein (1971), Kilambi (1975), Fenkart \& Binggeli (1979), Mermilliod (1981), Malysheva (1997), and Kharchenko et al. (2005). Complementary data related to spectroscopic or statistical studies, as well as trigonometric parallaxes and evolutionary models were performed by Nicolet (1981), Meynet et al. (1993), Loktin \& Beshenov (2001), Paunzen \& Netopil (2006), Piskunov et al. (2008), and Grosso \& Levato (2011). The reported true distance modulus is between $8.93 \mathrm{mag}$ and $10.15 \mathrm{mag}$, with distances from $614 \mathrm{pc}$ to $870 \mathrm{pc}$. The colour excess ranges from $0.08 \mathrm{mag}$ to $0.18 \mathrm{mag}$, 
Table 11. NGC 3114: Stellar fundamental parameters from the BCD system.

\begin{tabular}{|c|c|c|c|c|c|c|c|c|c|}
\hline $\begin{array}{c}\text { ID } \\
\text { Lynga }\end{array}$ & $\begin{array}{l}\text { Other } \\
\text { designation }\end{array}$ & $\begin{array}{c}D \\
{[\mathrm{dex}]}\end{array}$ & $\begin{array}{c}\lambda_{1} \\
[\AA]]\end{array}$ & $\begin{array}{l}\Phi_{\mathrm{b}} \text { S.T } \\
{[\mu]}\end{array}$ & $\begin{array}{l}T_{\text {eff }} \\
{[\mathrm{K}]}\end{array}$ & $\begin{array}{l}\log g \\
{[\mathrm{dex}]}\end{array}$ & $\begin{array}{c}M_{\mathrm{v}} \\
{[\mathrm{mag}]}\end{array}$ & $\begin{array}{c}M_{\mathrm{bol}} \\
{[\mathrm{mag}]}\end{array}$ & $\begin{array}{l}\Phi_{\mathrm{b}}^{0} \\
{[\mu]}\end{array}$ \\
\hline 003 & HD $87458^{d d}$ & 0.27 & 39 & $0.42{\text { B } 6 \mathrm{III}^{e}}^{e}$ & $15349 \pm 920$ & $3.18 \pm 0.28$ & $-1.90 \pm 0.57$ & $-2.83 \pm 0.60$ & $0.78 \pm 0.01$ \\
\hline 004 & $\mathrm{HD} 87241^{d d}$ & 0.38 & 37 & $1.13 \mathrm{~B}^{2} \mathrm{III}^{p, e}$ & $12250 \pm 506$ & $3.10 \pm 0.28$ & $-0.99 \pm 0.41$ & $-1.89 \pm 0.57$ & $0.85 \pm 0.02$ \\
\hline 005 & HD 87266 & 0.18 & 47 & $0.62 \mathrm{~B}^{2} \mathrm{III}^{p}$ & $20521 \pm 1161$ & $3.61 \pm 0.34$ & $-2.51 \pm 0.55$ & $-4.04 \pm 0.43$ & $0.72 \pm 0.01$ \\
\hline 006 & HD $87137^{d d}$ & 0.38 & 53 & 0.73 B8IV & $11886 \pm 277$ & $4.02 \pm 0.21$ & $-0.04 \pm 0.30$ & $-0.86 \pm 0.26$ & $0.85 \pm 0.02$ \\
\hline 007 & HD 87042 & 0.46 & 100 & $0.78{\mathrm{~A} 0 \mathrm{~V}^{m k}}^{m k}$ & $9790 \pm 500^{a}$ & $4.14 \pm 0.30^{a}$ & $0.65 \pm 0.50^{a}$ & $0.35 \pm 0.50^{a}$ & $1.07 \pm 0.02^{m}$ \\
\hline 009 & HD 87222 & 0.19 & 47 & $0.71{\mathrm{~B} 3 \mathrm{IV}^{p}}^{p}$ & $20203 \pm 1245$ & $3.63 \pm 0.32$ & $-2.39 \pm 0.53$ & $-3.87 \pm 0.44$ & $0.72 \pm 0.01$ \\
\hline 010 & HD 87349 & 0.34 & 50 & $0.22 \mathrm{~B} 7 \mathrm{~V}$ & $13079 \pm 469$ & $3.91 \pm 0.23$ & $-0.39 \pm 0.32$ & $-1.16 \pm 0.34$ & $0.81 \pm 0.01$ \\
\hline 011 & HD $87031^{d d}$ & 0.37 & 52 & 0.87 B8IV & $12341 \pm 296$ & $4.01 \pm 0.21$ & $-0.17 \pm 0.31$ & $-0.94 \pm 0.25$ & $0.84 \pm 0.02$ \\
\hline 013 & HD? & 0.34 & 53 & 0.77 B7V & 533 & $4.03 \pm 0.20$ & $-0.28 \pm 0.27$ & -1.09 & $0.81 \pm 0.01$ \\
\hline 015 & HD 8 & 0.24 & 52 & $0.66 \mathrm{~B} 4 \mathrm{~V}$ & $908 \pm 630$ & $3.98 \pm 0.23$ & $-1.31 \pm 0.35$ & $-2.54 \pm 0.41$ & $0.75 \pm 0.01$ \\
\hline 028 & HD 8 & 0.31 & 43 & $0.70 \mathrm{~B}^{2} \mathrm{IV}^{B e}$ & $09 \pm 690$ & $3.60 \pm 0.30$ & $-1.12 \pm 0.45$ & $-1.91 \pm 0.50$ & $0.79 \pm 0.01$ \\
\hline 033 & HD 8 & 0.28 & 49 & $0.79 \mathrm{~B}^{2} \mathrm{IV}^{B e}$ & $12 \pm 445$ & $3.87 \pm 0.25$ & $-0.90 \pm 0.38$ & $-1.95=$ & $0.78 \pm 0.01$ \\
\hline 040 & $\mathrm{HD} 3$ & 0.43 & 66 & $0.25 \mathrm{~A} 0 \mathrm{~V}$ & 337 & $4.32 \pm 0.11:$ & $0.63 \pm 0.37:$ & $0.12 \pm 0.24:$ & $0.95 \pm 0.04$ \\
\hline 047 & HD & 0.21 & 74 & 0.30 B4VI: ${ }^{B e}$ & 66: & $4.35 \pm 0.05:$ & $-1.40 \pm 0.13:$ & $-2.74 \pm 0.28$ & $0.75 \pm 0.01$ \\
\hline 066 & $\mathrm{HI}$ & 0.32 & 56 & $0.94 \mathrm{~B} 7 \mathrm{~V}$ & $0 \pm 539$ & $4.13 \pm 0.15$ & $-0.28 \pm 0.20$ & $-1.24=$ & $0.80=$ \\
\hline 067 & & 0.40 & 59 & $1.05 \mathrm{~B} 9 \mathrm{~V}$ & 288 & $4.19 \pm$ & & -0.5 & $0.88=$ \\
\hline 079 & $\mathrm{HL}$ & 0.40 & 89 & $1.27 \mathrm{~A}_{1} \mathrm{~V}^{m k}$ & $00^{a}$ & $4.17 \pm 0.20^{a}$ & 0.9 & $0.73 \pm$ & $1.14 \pm$ \\
\hline 085 & & 0.48 & 73 & $0.34 \mathrm{~A} 2 \mathrm{~V}:$ & & $4.44 \pm$ & & & \\
\hline 088 & & 0.49 & 62 & $0.85 \mathrm{~A} 1 \mathrm{~V}$ & 68 & \pm 0.15 & 1.2 & 46: & 0.05 \\
\hline 091 & & 0.37 & 48 & $0.99 \mathrm{~B}^{2} \mathrm{IV}^{s}$ & $12269 \pm 311$ & $3.82 \pm$ & $-0.40 \pm 0.35$ & .37 & 0.02 \\
\hline 097 & & 0.37 & 60 & $0.79 \mathrm{~B}$ & 318 & 4.2 & & 24 & .02 \\
\hline 099 & $\mathrm{CP}$ & 0.41 & 59 & 1.1 & 272 & 15 & & 25: & 03 \\
\hline 100 & & 0 & 74 & 0.9 & 318 & 4.5 & 36: & -0 & 03 \\
\hline 102 & & 0.40 & 60 & $1.36 \mathrm{~B}$ & 328 & 4.20 & 32 & -0.45 & 0.8 \\
\hline 105 & & 0 & 74 & $1.08 \mathrm{~A}$ & & & & 0.2 & \\
\hline 124 & & 34 & 59 & $0.98 \mathrm{~B}$ & 87 & 5 & 26 & -0 & 0 . \\
\hline 126 & & 9 & 52 & $1 .($ & & & -0 & -0 & \\
\hline 127 & & 0 & 55 & $1 .($ & & 4.0 & & -0 & \\
\hline 129 & & 0.35 & 55 & 0. & 64 & 4. & 24 & -( & \\
\hline 130 & & 7 & 82 & 1.3 & $00^{a}$ & 4.2 & & & 1.2 \\
\hline 132 & $\mathrm{HD} ?$ & 0.29 & 72 & $1 .($ & 48 & 4.4 & -0 & -1 . & $0.81=$ \\
\hline 134 & & 0.34 & 73 & $0.96 \mathrm{~B}$ & & & $24:$ & -0 & 0.8 \\
\hline 136 & & 0.32 & 50 & $0.97 \mathrm{~B}$ & & & & -1 & 0.8 \\
\hline 141 & HD & 0.16 & 62 & $0.85 \mathrm{~B}$ & $22033 \pm$ & $4.15 \pm 0$ & $-1.93 \pm$ & -3.76 & $0.71 \pm 0.00$ \\
\hline 147 & & 0.42 & 58 & $1.01 \mathrm{~B}$ & & $4.13 \pm 0$ & $0.46 \pm$ & $-0.44=$ & $0.90 \pm 0.02$ \\
\hline 149 & $\mathrm{HD} \&$ & 0.41 & 47 & $0.91 \mathrm{~B} 9 \mathrm{IV}$ & $650 \pm 231$ & $3.80 \pm 0.27$ & $-0.29 \pm 0.36$ & $-0.99 \pm$ & $0.87 \pm 0.02$ \\
\hline 186 & HD 8 & 0.30 & 69 & 0.73 B7VI: & $851 \pm 543$ & $4.37 \pm 0.08$ & $-0.70 \pm 0.19$ & $-1.31 \pm 0.17$ & $0.81 \pm 0.02$ \\
\hline$\ldots$ & HD 3 & 0.41 & 63 & $1.09 \mathrm{~B} 9 \mathrm{~V}$ & $11155 \pm 301$ & $4.27 \pm 0.14$ & $0.53 \pm 0.44:$ & $0.03 \pm 0.30^{f}$ & $0.91 \pm 0.03$ \\
\hline$\ldots$ & HD 8 & 0.53 & 50 & $1.30 \mathrm{~A} 7 \mathrm{III}$ & $7550 \pm 500^{b v}$ & $3.05 \pm 0.30^{a}$ & $-0.19 \pm 0.50^{f}$ & $-0.16 \pm 0.50^{a}$ & $1.79 \pm 0.02^{m}$ \\
\hline$\cdots$ & & 0.16 & 52 & $0.67 \mathrm{~B} 2 \mathrm{IV}^{B e}$ & 127 & $3.87 \pm 0.28$ & $-2.43 \pm$ & -4.16 & $0.71 \pm 0.00$ \\
\hline$\cdots$ & $\mathrm{HL}$ & 0.31 & 56 & $0.97 \mathrm{~B} 6 \mathrm{~V}$ & & $4.15 \pm 0.14$ & $-0.30 \pm$ & -1.2 & $0.80 \pm 0.01$ \\
\hline$\ldots$ & CPD & 0.37 & 46 & 0.86 B8IV & 297 & $3.78 \pm 0.28$ & $-0.53 \pm 0.37$ & $-1.23 \pm$ & $0.83 \pm 0.02$ \\
\hline$\cdots$ & J10015881-6001125 & 0.41 & 55 & $0.60 \mathrm{~B} 9 \mathrm{~V}$ & $11371 \pm 277$ & $4.05 \pm 0.18$ & $0.20 \pm 0.29$ & $-0.64 \pm 0.29$ & $0.88 \pm 0.02$ \\
\hline
\end{tabular}

Notes. Nomenclature according to Lyngå (1962). The symbol : is used to indicate extrapolated values. $g$ is the stellar surface gravity given in $\mathrm{cm} \mathrm{s}^{-2} .{ }^{(d d)}$ Stars with a double Balmer discontinuity. ${ }^{(m k)}$ Spectral type determined with the MK method. ${ }^{(B e)}$ Known Be stars. ${ }^{(e, s h e l l)}$ Reported by González \& Lapasset (2001). ${ }^{(p)}$ Reported as peculiar stars in the literature. ${ }^{(B o o)}$ Star reported as $\lambda$ Boothis variable by Schneider \& Weiss (1988). ${ }^{(a)}$ Values interpolated from Cox (2000). ${ }^{(b v)} T_{\text {eff }}$ values from Boehm-Vitense (1982). ${ }^{(f)} M_{\text {bol }}$ values estimated with the bolometric correction given by Flower (1996). ${ }^{(m)}$ Values determined using the relation between $\Phi_{\mathrm{b}}$ and $(B-V)$ given by Moujtahid et al. (1998).

and the age, which is more uncertain, is between $71 \mathrm{Myr}$ and 126 Myr.

Regarding spectral classification, all stars of our sample were previously classified with the exception of No. 86 (see Table 4). Star No. 1 is a known Be star, No. 6 is reported as Be star by Grosso \& Levato (2011), Nos. 11 and 24 are line peculiar stars, and No. 90 is a chemically peculiar star. In addition, the star No. 26 is a known eclipsing binary and No. 12 is reported as a variable star.

The derived BCD parameters of each star are quoted in Table 13 while their corresponding physical parameters, distances and ages are given in Table 14. From the values of the last table, we calculate a mean true distance modulus
$\overline{\left(m_{\mathrm{v}}-M_{\mathrm{v}}\right)_{0}}=9.25 \pm 0.2 \mathrm{mag}$, which corresponds to $\langle d\rangle=710 \pm$ $80 \mathrm{pc}$, a mean distance $\bar{d}=746 \pm 144 \mathrm{pc}$ and a mean colour excess $\overline{E(B-V)}=0.34 \pm 0.02 \mathrm{mag}$. In addition, we construct a well-defined HR diagram using the BCD parameters (Fig. 7). Considering then that stars No. 1 (Mermilliod 1982b) and No. 12 (see below) are blue stragglers, the cluster age would be between $40 \mathrm{Myr}$ and $63 \mathrm{Myr}$. This value agrees with the mean age, $\bar{t}=68 \pm 13 \mathrm{Myr}$, derived by averaging the individual stellar estimates.

Our distance and age estimates agree very well with the value reported by Paunzen \& Netopil (2006, $725 \pm 94$ pc), Malysheva (1997, 71 Myr), and Paunzen \& Netopil (2006, 74 Myr). 


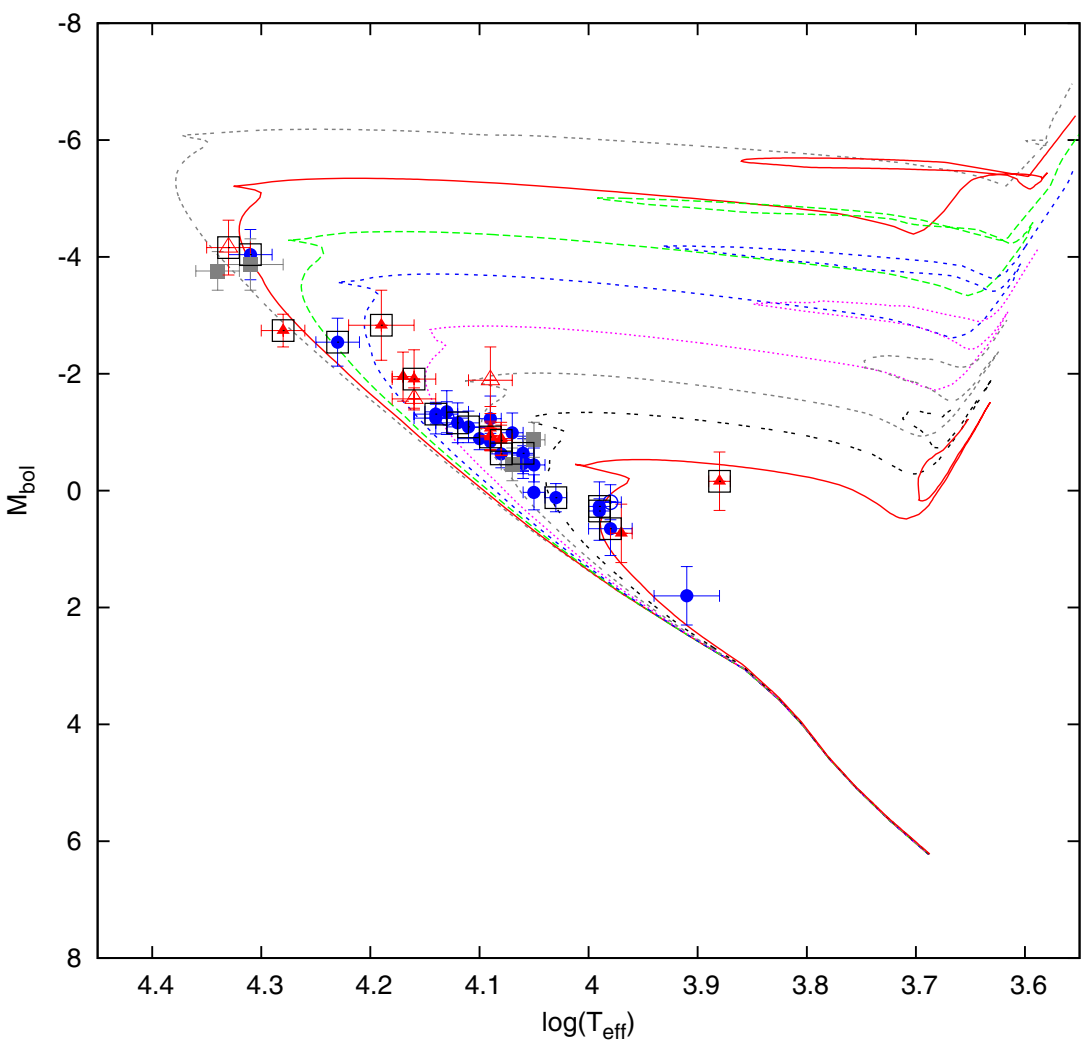

Fig. 6. NGC 3114: HR diagram. The isochrone curves are given by Ekström et al. (2012). Probable members of the clusters are denoted in $\bullet$ (blue) symbols, pnm in $\odot$ (blue), and non-members $(\mathrm{nm})$ in $\mathbf{~ ( g r e y ) . ~}$ Be star cluster members are indicated in $\Delta$ (red) and Be pnm in $\triangle$ (red). The stars denoted in $\square$ symbols have a negative colour gradient excess.
Our HR diagram shows a lack of stars with $M_{\text {bol }}$ between 0 and 1 mag (as was reported by Kilambi 1975; Mermilliod 1981), but to confirm the existence of the gap we need to observe a substantially larger sample.

The star No. 86 has been classified for first time as A2VI. The BCD spectral classification of the rest of sample agrees with the classification done, mainly, by Grosso \& Levato (2011). Stars Nos. 1 and 6 are variable stars because the former showed a $\mathrm{H} \beta$ absorption line profile with a double-peaked emission core (Grosso \& Levato 2011), but our spectrum shows an absorption line with a central emission core. In the same work the latter was also reported with an emission in the $\mathrm{H} \beta$ line, but we observe it as a normal B-type star (see Fig. 2).

Stars Nos. 3 and 25 are certainly Be stars because they display a second Balmer discontinuity in their spectra. The former is a fast rotator (Grosso \& Levato 2011, $240 \mathrm{~km} \mathrm{~s}^{-1}$ ).

Stars Nos. 8, 11, 14, 15, 26, 43, 86, and 90 were reported as non-members (Feinstein 1971; Kilambi 1975; Grosso \& Levato 2011) and stars Nos. 6 and 26 as pnm by Grosso \& Levato (2011). According to our membership criterion, we find that stars Nos. 11, 15, and 26 are non-members, but stars Nos. 8, $14,43,86$, and 90 could be cluster members. In addition, we conclude that star No. 23 is non-member and No. 12 is a pnm.

The only star reported by Mermilliod (1982b) as blue straggler is No. 1. Our HR diagram confirms this finding. Although we classified No. 12 as a pnm, this object could be a blue straggler on account of the uncertainties in the measurements.

\section{Discussion}

As mentioned above, roughly $50 \%$ of the studied stellar sample in NGC 3114 has a clear sign of an anomalous colour-gradient excess, i.e. the index $\Phi_{\mathrm{b}}^{\mathrm{obs}}-\Phi_{\mathrm{b}}^{0}\left(\lambda_{1}, D\right)$ is negative indicating that there is a high unexpected blueing.
This negative colour index cannot be attributed either to uncertainties affecting the measurements or to reduction techniques. Along different observing runs, stars of this cluster were observed together with flux standards and stars belonging to other clusters. In addition, we applied the same reduction procedure to all of them. However, stars with negative colourgradient excesses were found in just one star belonging to NGC 2645 (No. 56) and in half of the studied sample of NGC 3114.

There are at least three obvious possibilities to account for this phenomenon: changes in the colour gradients due to anomalous stellar metallicity, effects induced by the rapid rotation of stars, and binarity.

Metallicity effects: according to Chalonge \& Divan (1977), negative values in $\left(\Phi_{b}-\Phi_{b}^{0}\right)$ are typically found in late-type poor metal stars. In early-type stars this phenomenon does not seem to be relevant. It is also known that chemically peculiar stars are affected by the concomitant blanketing effect (cf. Cidale et al. 2007) and present a smaller Balmer jump if compared to normal stars showing the same near-infrared flux (Leone \& Catalano 1991). Particularly, NGC 3114 has many Am and Ap stars, nevertheless, using models of stellar atmospheres calculated for metallicities other than solar, we realize that the obtained blueing does not attain the amount reported in Table 11.

Rapid-rotation effects: rapid-rotation produces an external oblateness of stars that induces a variation with latitude of the surface gravity and the local effective temperature, known as the gravitational darkening effect (GDE, von Zeipel 1924). Because of the GDE, surface gravities and effective temperatures deduced with models or calibrations based on plane-parallel or axisymmetric stellar atmospheres should be considered apparent and dependent on the inclination angle between the stellar rotation axis and 


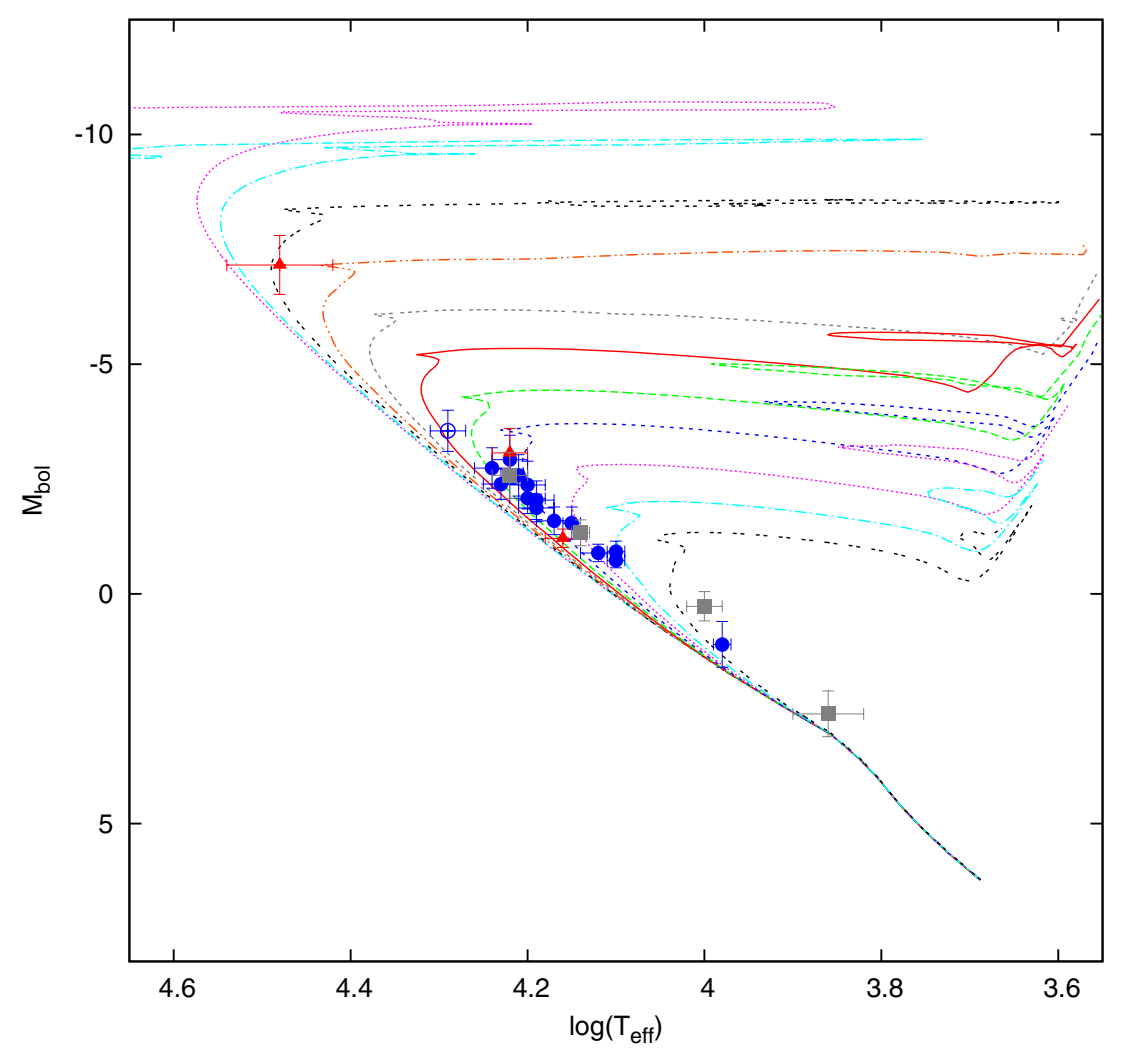

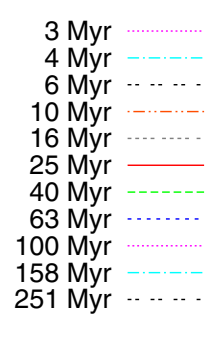

Fig. 7. NGC 6025: HR diagram. The estimated age of this cluster is in between 40 and $69 \mathrm{Myr}$. The isochrone curves are given by Ekström et al. (2012). Probable members of the clusters are denoted in • (blue) symbols, pnm in $\odot$ (blue), and non-members (nm) in - (grey). Be star cluster members are indicated in $\boldsymbol{\Delta}$ (red). the sight direction (cf. Maeder \& Peytremann 1970; Collins et al. 1991; Frémat et al. 2005; Torres \& Cidale 2007). In NGC 3114, there are a large number of Be stars as revealed by the presence of the second Balmer discontinuity. This large number of Be stars might imply that there may also be a significant number of rapid rotators that momentarily do not display the Be phenomenon or have not attained the required rotational properties for being considered Be stars, but they unavoidable have spectra affected by the GDE, which leads to misleading interpretations of their $\left(\Phi_{\mathrm{b}}-\Phi_{\mathrm{b}}^{0}\right)$ colour gradient. Nevertheless, looking at the tables of $(B-V)$ colour indices for rapid rotators published by Collins et al. (1991), we see that rotation can introduce some blueing effects. Apart from this, we note that one third of stars reported in Table 3 are Be stars where the (free-free) and (bound-free) radiation of the circumstellar envelope may significantly change the $\left(\Phi_{\mathrm{b}}-\Phi_{\mathrm{b}}^{0}\right)$ colour index. It can be shown that compact and hot circumstellar envelopes can produce apparent blueings (Moujtahid et al. 1998, 1999).

Binarity: the number of unresolved binaries in clusters can be quite significant. The binarity hypothesis is supported by the fact that it is thought to be responsible for chemical anomalies in the CP group (cf. Stateva et al. 2012; Quiroga et al. 2010). Binary systems evolve at short timescales. Because of the evolution effects, one of the companions can become redder, originating changes in the colour gradients. At rather small differences between the luminosities of both components, it is possible to have a Balmer discontinuity mainly reflecting the cooler component, while the energy distribution in the visual spectral region is dominated by the hotter component. This can lead to a high negative value of $\left(\Phi_{\mathrm{b}}^{\mathrm{obs}}-\Phi_{\mathrm{b}}^{0}\right)$. Moreover, apart from the oblateness of components in close binary systems and the concomitant
GDE induced by the mutual gravity-rotation interaction, there is also a brightening of the stellar hemispheres in components that face each other, which can produce peculiar spectra (Palate et al. 2013) and increase the blueing effect. Therefore, on the one hand, the Be phenomenon, and on the other hand, uncovered binarity, could account for the reported negative colour gradient-excesses.

We also call the attention to the fact that stars with $-1<$ $M_{\text {bol }}<1$ are significantly detached from the main sequence towards redder colours, so that it becomes difficult to estimate the cluster age. That is the case for NGC 3114 and Collinder 223. Although, the BCD parameters are expected to be free from extinction effects, the fact that some stars in NGC 3114 have observed colour gradients that are bluer than the intrinsic gradient for a star with the same physical parameters might suggest that many other stars are also anomalous (with gradients that are close to the intrinsic gradient). As a consequence, the underestimation of the cluster reddening, produced by the spectral anomalies of the stars in the cluster, also leads us to overestimates of the distance modulus and stellar luminosities. A similar discussion can be applied to Collinder 223, although it is without detected spectral anomalies because of its larger distance modulus and a larger ISM reddening. In this cluster, it is apparent that all member and probable non-member stars are placed distinctly above the main sequence (see Fig. 3). This fact could explain the difficulties we found in estimating the cluster age.

Another possibility that cannot be ruled out is the presence of an incipient double turn-off associated with the population of field stars. In fact, noticeable evidences of a secondary turn-off was revealed in two old LMC clusters: NGC 1848 (Mackey \& Broby Nielsen 2007) and NGC 1868 (Santiago et al. 2002), which differ in age by $\sim 300 \mathrm{Myr}$. For this behaviour, the first authors proposed that both clusters could be formed in a 
Table 12. NGC 3114: Physical parameters and distance to stars.

\begin{tabular}{|c|c|c|c|c|c|c|c|c|}
\hline $\begin{array}{c}\text { ID } \\
\text { Lynga }\end{array}$ & $\begin{array}{l}\text { Other } \\
\text { designation }\end{array}$ & $\begin{array}{c}m_{\mathrm{v}} \\
{[\mathrm{mag}]}\end{array}$ & $E(B-V)$ & $\begin{array}{c}\left(m_{\mathrm{v}}-M_{\mathrm{v}}\right)_{0} \\
{[\mathrm{mag}]}\end{array}$ & $\begin{array}{c}d \\
{[\mathrm{pc}]}\end{array}$ & $\begin{array}{l}\log \mathcal{L} \\
{\left[\mathcal{L}_{\odot}\right]}\end{array}$ & $\begin{array}{c}M \\
{\left[M_{\odot}\right]}\end{array}$ & $\begin{array}{c}\text { Age } \\
{[\mathrm{Myr}]}\end{array}$ \\
\hline 003 & $\mathrm{HD} 87458^{d d}$ & 7.73 & . & 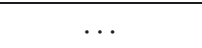 & $\cdots$ & $3.03 \pm 0.24$ & $6.91 \pm 2.06$ & $51 \pm 22$ \\
\hline 004 & HD $87241^{d d}$ & 7.77 & $0.21 \pm 0.02$ & $8.10 \pm 0.4^{p n m}$ & $418 \pm 79$ & $2.65 \pm 0.23$ & $5.24 \pm 1.49$ & $102 \pm 50$ \\
\hline 005 & HD 87266 & 8.23 & $\ldots$ & $\ldots$ & $\ldots$ & $3.51 \pm 0.17$ & $8.74 \pm 1.71$ & $23 \pm 6$ \\
\hline 006 & HD $87137^{d d}$ & 8.29 & $\ldots$ & $\ldots$ & $\ldots$ & $2.24 \pm 0.10$ & $3.33 \pm 0.21$ & $165 \pm 31$ \\
\hline 007 & HD 87042 & 8.36 & $\ldots$ & $\ldots$ & $\ldots$ & $1.76 \pm 0.20$ & $2.46 \pm 0.24$ & $294 \pm 145$ \\
\hline 009 & HD 87222 & 8.63 & $0.00 \pm 0.01$ & $11.02 \pm 0.5^{n m}$ & $1596 \pm 395$ & $3.44 \pm 0.18$ & $8.37 \pm 1.55$ & $24 \pm 7$ \\
\hline 010 & HD 87349 & 8.62 & $\ldots$ & $\ldots$ & $\ldots$ & $2.36 \pm 0.14$ & $3.84 \pm 0.31$ & $117 \pm 17$ \\
\hline 011 & HD $87031^{d d}$ & 8.73 & $0.03 \pm 0.01$ & $8.82 \pm 0.3$ & $580 \pm 83$ & $2.27 \pm 0.10$ & $3.48 \pm 0.22$ & $144 \pm 24$ \\
\hline 013 & HD 304848 & 9.19 & $\ldots$ & $\ldots$ & $\ldots$ & $2.33 \pm 0.11$ & $3.65 \pm 0.24$ & $118 \pm 27$ \\
\hline 015 & HD 87612 & 9.72 & $\ldots$ & $\ldots$ & $\ldots$ & $2.91 \pm 0.16$ & $5.47 \pm 0.44$ & $42 \pm 11$ \\
\hline 028 & HD $87404^{d d}$ & 8.96 & $\ldots$ & $\ldots$ & $\ldots$ & $2.66 \pm 0.20$ & $4.90 \pm 0.77$ & $80 \pm 18$ \\
\hline 033 & HD $87818^{d d}$ & 9.41 & $0.01 \pm 0.01$ & $10.28 \pm 0.4^{p n m}$ & $1136 \pm 198$ & $2.68 \pm 0.17$ & $4.71 \pm 0.41$ & $72 \pm 10$ \\
\hline 040 & HD 304894 & 10.35 & $\ldots$ & $\ldots$ & $\ldots$ & $1.85 \pm 0.10$ & $2.58 \pm 0.18$ & $146 \pm 114$ \\
\hline 047 & $\mathrm{HD} 87565^{d d}$ & 9.67 & $\ldots$ & $\ldots$ & $\ldots$ & $2.99 \pm 0.11$ & $5.69 \pm 0.37$ & $6 \pm 9$ \\
\hline 058 & CPD-59 1748 & 10.49 & $\ldots$ & $\ldots$ & $\ldots$ & $1.79 \pm 0.05$ & $2.30 \pm 0.28$ & $194 \pm 192$ \\
\hline 066 & HD 304841 & 10.15 & $0.11 \pm 0.01$ & $10.10 \pm 0.2^{p m m}$ & $1048 \pm 99$ & $2.39 \pm 0.11$ & $3.76 \pm 0.20$ & $88 \pm 32$ \\
\hline 067 & HD 304840 & 9.20 & $0.12 \pm 0.02$ & $8.45 \pm 0.3$ & $491 \pm 62$ & $2.11 \pm 0.10$ & $3.02 \pm 0.18$ & $151 \pm 68$ \\
\hline 079 & HD 30 & 9.74 & $0.10 \pm 0.02$ & $8.45 \pm 0.5$ & $490 \pm 114$ & $1.60 \pm 0.20$ & $2.28 \pm 0.18$ & $345 \pm 172$ \\
\hline 088 & HD 304837 & 10.18 & $\ldots$ & $\cdots$ & $\ldots$ & $1.64 \pm 0.18$ & $2.35 \pm 0.15$ & $336 \pm 121$ \\
\hline 091 & HD $87125^{d d}$ & 8.56 & $0.11 \pm 0.02$ & $8.61 \pm 0.3$ & $526 \pm 84$ & $2.32 \pm 0.15$ & $3.67 \pm 0.31$ & $152 \pm 15$ \\
\hline 097 & HD 87205 & 9.43 & $\ldots$ & $\ldots$ & $\ldots$ & $2.15 \pm 0.10$ & $3.13 \pm 0.18$ & $121 \pm 62$ \\
\hline 099 & CPD-59 1677 & 10.02 & $0.16 \pm 0.02$ & $9.06 \pm 0.3$ & $650 \pm 87$ & $2.08 \pm 0.10$ & $2.97 \pm 0.18$ & $158 \pm 70$ \\
\hline 100 & HD 304836 & 11.06 & $0.03 \pm 0.02$ & $11.35 \pm 0.4^{n m}$ & $1861 \pm 306$ & $2.24 \pm 0.12$ & $2.79 \pm 0.50$ & $114 \pm 114$ \\
\hline 102 & HD 304835 & 9.07 & $0.36 \pm 0.02$ & $7.56 \pm 0.3^{n m}$ & $325 \pm 49$ & $2.08 \pm 0.11$ & $3.00 \pm 0.17$ & $144 \pm 67$ \\
\hline 105 & CPD-59 1666 & 11.04 & $0.01 \pm 0.04$ & $10.66 \pm 0.4^{p n m}$ & $1356 \pm 254$ & $1.82 \pm 0.12$ & $2.30 \pm 0.32$ & $237 \pm 195$ \\
\hline 124 & HD 304854 & 9.84 & $0.12 \pm 0.02$ & $9.52 \pm 0.3$ & $800 \pm 96$ & $2.25 \pm 0.08$ & $3.36 \pm 0.19$ & $100 \pm 51$ \\
\hline 126 & HD $87309^{d d}$ & 9.31 & $0.14 \pm 0.01$ & $8.92 \pm 0.3$ & $607 \pm 92$ & $2.25 \pm 0.11$ & $3.38 \pm 0.22$ & $168 \pm 17$ \\
\hline 127 & HD 304855 & 9.87 & $0.13 \pm 0.02$ & $9.24 \pm 0.3$ & $705 \pm 86$ & $2.14 \pm 0.11$ & $3.11 \pm 0.18$ & $176 \pm 47$ \\
\hline 129 & HD $87366^{d d}$ & 9.42 & $\ldots$ & $\ldots$ & $\ldots$ & $2.26 \pm 0.10$ & $3.39 \pm 0.18$ & $130 \pm 41$ \\
\hline 130 & HD 304856 & 11.13 & $0.13 \pm 0.04$ & $8.78 \pm 0.5$ & $569 \pm 132$ & $1.18 \pm 0.20$ & $1.82 \pm 0.15$ & $484 \pm 369$ \\
\hline 132 & HD $304831^{d d}$ & 9.74 & $0.15 \pm 0.01$ & $10.10 \pm 0.2^{p n m}$ & $1049 \pm 93$ & $2.52 \pm 0.08$ & $3.82 \pm 0.33$ & $41 \pm 43$ \\
\hline 134 & HD 87567 & 9.60 & $0.07 \pm 0.02$ & $10.07 \pm 0.2$ & $1031 \pm 112$ & $2.23 \pm 0.06$ & $3.10 \pm 0.32$ & $75 \pm 76$ \\
\hline 136 & HD 87581 & 9.24 & $0.13 \pm 0.01$ & $9.38 \pm 0.4$ & $750 \pm 122$ & $2.44 \pm 0.14$ & $4.07 \pm 0.35$ & $99 \pm 15$ \\
\hline 141 & HD 304832 & 9.82 & $0.09 \pm 0.01$ & $11.46 \pm 0.3^{\mathrm{nm}}$ & $1955 \pm 275$ & $3.40 \pm 0.13$ & $7.82 \pm 0.51$ & $9 \pm 6$ \\
\hline 147 & HD 304858 & 9.54 & $0.08 \pm 0.02$ & $8.83 \pm 0.2^{p n m}$ & $584 \pm 65$ & $2.07 \pm 0.12$ & $2.96 \pm 0.17$ & $190 \pm 58$ \\
\hline 149 & HD 87507 & 8.48 & $0.03 \pm 0.01$ & $8.68 \pm 0.4$ & $544 \pm 91$ & $2.29 \pm 0.14$ & $3.49 \pm 0.26$ & $183 \pm 17$ \\
\hline 186 & HD 86981 & 9.72 & $\ldots$ & $\ldots$ & $\ldots$ & $2.42 \pm 0.07$ & $3.64 \pm 0.27$ & $46 \pm 45$ \\
\hline$\ldots$ & HD 304838 & 9.87 & $0.13 \pm 0.02$ & $8.94 \pm 0.4$ & $613 \pm 125$ & $1.88 \pm 0.12$ & $2.73 \pm 0.15$ & $137 \pm 86$ \\
\hline$\ldots$ & HD $87436^{d d}$ & 6.19 & $\ldots$ & $\ldots$ & $\ldots$ & $1.96 \pm 0.20$ & $3.10 \pm 0.67$ & $368 \pm 167$ \\
\hline$\cdots$ & HD 87801 & 9.10 & $\ldots$ & $\cdots$ & $\cdots$ & $3.56 \pm 0.19$ & $8.58 \pm 1.02$ & $18 \pm 6$ \\
\hline$\ldots$ & HD 304839 & 9.20 & $0.13 \pm 0.01$ & $9.10 \pm 0.2$ & $661 \pm 74$ & $2.39 \pm 0.11$ & $3.83 \pm 0.19$ & $75 \pm 31$ \\
\hline$\ldots$ & CPD-59 1691 & 8.78 & $0.02 \pm 0.01$ & $9.24 \pm 0.4$ & $704 \pm 120$ & $2.39 \pm 0.16$ & $3.77 \pm 0.32$ & $149 \pm 16$ \\
\hline$\ldots$ & $\mathrm{J} 10015881-6001125$ & $\cdots$ & $\cdots$ & $\cdots$ & $\ldots$ & $2.15 \pm 0.12$ & $3.12 \pm 0.19$ & $194 \pm 35$ \\
\hline
\end{tabular}

Notes. $m_{\mathrm{v}}$ values obtained from SIMBAD database. ${ }^{(d d)}$ Stars with double Balmer discontinuity. ${ }^{(n m)}$ Non-member stars. ${ }^{(p n m)}$ Probable non-member stars.

single giant molecular cloud but were captured into a common cluster group by tidal interaction. This scenario can naturally account for differences in age and spatial distributions, as well as the uniform metallicity. It is interesting to stress that both clusters (NGC 3114 and Collinder 223) also have a significant internal spread of stellar distances, which might support the scenario of tidal interaction or capture.

An alternative hypothesis that might lead to a significantly detached number of stars from the main sequence towards redder colours is the unresolved binary nature of stars. Both luminosity shifts of about $0.75 \mathrm{mag}$, attributed to the presence of companions of similar luminosity, where the secondary became redder through evolution effects, could be responsible for the observed bump in the HR diagrams. However, as this phenomenon is not observed in all clusters, the incidence of binarity vs. spectral type should be explored more thoroughly in open clusters. Finally, it should be mentioned that because of rotational effects, the apparent BCD parameters have lead to the identification of lower $T_{\text {eff }}$ and $\log g$ values as the stars are more evolved and shift to the red side of the HR diagram (Zorec et al. 2005).

\section{Conclusions}

The BCD spectrophotometric method is a powerful and easy-touse tool to determine fundamental physical parameters of earlytype stars, making it adequate to explore the properties of stellar clusters. The knowledge of accurate values of effective temperature, surface gravity, stellar mass, and luminosity of stars in open 
Table 13. NGC 6025: Stellar fundamental parameters from the BCD system.

\begin{tabular}{|c|c|c|c|c|c|c|c|c|c|c|}
\hline $\begin{array}{c}\text { ID } \\
\text { Feinstein } \\
\text { Kilambi }\end{array}$ & $\begin{array}{l}\text { Other } \\
\text { designation }\end{array}$ & $\begin{array}{c}D \\
{[\operatorname{dex}]}\end{array}$ & $\begin{array}{c}\lambda_{1} \\
[\AA]]\end{array}$ & $\begin{array}{c}\Phi_{\mathrm{b}} \\
{[\mu]}\end{array}$ & S.T & $\begin{array}{l}T_{\text {eff }} \\
{[\mathrm{K}]}\end{array}$ & $\begin{array}{l}\log g \\
{[\operatorname{dex}]}\end{array}$ & $\begin{array}{c}M_{\mathrm{v}} \\
{[\mathrm{mag}]}\end{array}$ & $\begin{array}{c}M_{\mathrm{bol}} \\
{[\mathrm{mag}]}\end{array}$ & $\begin{array}{l}\Phi_{\mathrm{b}}^{0} \\
{[\mu]}\end{array}$ \\
\hline 01 & 8 & 6 & 81 & 1.20 & $\mathrm{~B}$ & $0000 \pm 4275^{a}$ & $3.94 \pm 0.00^{a}$ & $-4.00 \pm 0.64^{a}$ & $-7.16 \pm 0.64^{a}$ & $.68 \pm 0.01$ \\
\hline 02 & HD 1 & 0.26 & 47 & 1.29 & B5IV & $16275 \pm 531$ & $3.78 \pm 0.29$ & $-1.49 \pm 0.47$ & $-2.58 \pm 0.45$ & $0.76 \pm 0.01$ \\
\hline 03 & HD $143413^{d d}$ & 0.24 & 43 & 1.21 & B5III & 16780 & $3.46 \pm 0.32$ & $-1.87 \pm 0.55$ & $-3.07 \pm 0.53$ & 0.76 \\
\hline 04 & CD-60 6021 & 0.34 & 57 & 1.20 & B7V & $12857=$ & 11 & $-0.13 \pm$ & $-0.92 \pm 0.23$ & $82 \pm 0.02$ \\
\hline 05 & 6007 & 0.36 & 61 & 1.17 & B7V & 76 & $3 \pm 0.13$ & $3 \pm 0$ & $-0.73 \pm$ & 0.02 \\
\hline 06 & 288 & 0.29 & 57 & 1.42 & $\mathrm{~B} 6 \mathrm{~V}^{e}$ & $50=$ & $9 \pm 0.14$ & $45 \pm$ & $-1.59 \pm$ & $0.78 \pm$ \\
\hline 07 & HD 1 & 0.24 & 44 & 1.36 & B5III & $607=$ & $9 \pm$ & 55 & -2.92 & $0.76 \pm$ \\
\hline 08 & CD-606015 & 0.27 & 53 & 1.17 & B5V & $15705 \pm$ & $4.04 \pm 0.19$ & $-0.84 \pm$ & $-2.08 \pm 0.33$ & $0.77 \pm 0.01$ \\
\hline 11 & HD 143287 & 0.31 & 55 & 1.23 & $\mathrm{~B}_{6} \mathrm{~V}^{p}$ & $13805 \pm 522$ & $4.11 \pm 0.17$ & $-0.35 \pm 0.20$ & $-1.33 \pm 0.28$ & $0.80 \pm 0.01$ \\
\hline 12 & HD 143309 & 0.20 & 48 & 1.23 & $\mathrm{~B}^{2} \mathrm{IV}^{v}$ & $19386 \pm 1081$ & $3.70 \pm 0.32$ & $-2.19 \pm 0.51$ & $-3.55 \pm 0.45$ & $0.73 \pm 0.01$ \\
\hline 13 & CD-60 6011 & 0.28 & 49 & 0.97 & B5IV & $15558 \pm$ & $3.85 \pm 0.26$ & $-0.93 \pm 0.41$ & $-2.04 \pm 0.42$ & $0.77 \pm 0.01$ \\
\hline 14 & CD-606013 & 27 & 55 & 1.07 & B6V & $75 \pm$ & $4.10 \pm 0.17$ & $-0.74 \pm 0.28$ & $-1.87 \pm 0.30$ & $0.77 \pm 0.01$ \\
\hline 15 & CD-60 6017 & 0.31 & 77 & 2.23 & F0V: & $7300 \pm 700^{a}$ & $4.34 \pm 0.50^{a}$ & $2.70 \pm 0.50^{a}$ & $2.61 \pm 0.50^{a}$ & $1.67 \pm 0.03$ \\
\hline 16 & CD-60 6027 & 0.31 & 51 & 1.38 & B6V & $14017 \pm 453$ & $3.97 \pm 0.22$ & $-0.57 \pm 0.34$ & $-1.54 \pm 0.35$ & $0.79 \pm 0.01$ \\
\hline 22 & HD 143388 & 0.24 & 50 & 1.18 & B4IV & $17379 \pm 692$ & $3.87 \pm 0.26$ & $-1.46 \pm 0.41$ & $-2.74 \pm 0.44$ & $0.75 \pm 0.01$ \\
\hline 23 & CD-60 6024 & 0.25 & 48 & 1.29 & B4IV & $16696 \pm 659$ & $3.81 \pm 0.28$ & $-1.45 \pm 0.43$ & $-2.58 \pm 0.50$ & $0.76 \pm 0.01$ \\
\hline 24 & CPD-596562 & 0.34 & 60 & 1.39 & B7V & $13090 \pm 490$ & $4.23 \pm 0.13$ & $0.03 \pm 0.31$ & $-0.89 \pm 0.19$ & $0.83 \pm 0.02$ \\
\hline 25 & HD $143412^{d d}$ & 0.31 & 59 & 1.39 & $\mathrm{~B} 7 \mathrm{~V}^{p}$ & $14316 \pm 714$ & $4.22 \pm 0.13$ & $-0.26 \pm 0.25$ & $-1.21 \pm 0.20$ & $0.80 \pm 0.01$ \\
\hline 26 & V 396 Nor & 0.46 & 70 & 1.55 & $\mathrm{~A} 1 \mathrm{~V}^{E B}$ & $9938 \pm 376$ & $4.39 \pm 0.10:$ & $0.52 \pm 0.22:$ & $0.27 \pm 0.32^{a}$ & $1.02 \pm 0.04$ \\
\hline 43 & HD $14312 ?$ & 0.25 & 53 & 1.40 & B4V & $17010 \pm 770$ & $4.02 \pm 0.21$ & $-1.08 \pm 0.36$ & $-2.39 \pm 0.33$ & $0.76 \pm 0.01$ \\
\hline 86 & CPD-60 6287 & 0.48 & 79 & 1.60 & A2:VI: & $9540 \pm 318:$ & $4.20 \pm 0.30^{a}$ & $1.30 \pm 0.50^{a}$ & $1.10 \pm 0.50^{a}$ & $1.12 \pm 0.05$ \\
\hline 90 & HD 143027 & 0.27 & 45 & 1.46 & $\mathrm{~B} 5 \mathrm{IV}^{p}$ & $15705 \pm 583$ & $3.66 \pm 0.29$ & $-1.41 \pm 0.48$ & $-2.37 \pm 0.52$ & $0.77 \pm 0.01$ \\
\hline
\end{tabular}

Notes. Nomenclature according to Feinstein (1971) and Kilambi (1975). The symbol : is used to indicate extrapolated values. $g$ is the stellar surface gravity given in $\mathrm{cm} \mathrm{s}^{-2}$. ${ }^{(d d)}$ Stars with double Balmer discontinuity. ${ }^{(B e)}$ Known Be stars. ${ }^{(e)} \mathrm{H} \beta$ in emission, reported by Grosso \& Levato (2011). ${ }^{(p)}$ Reported as peculiar star. ${ }^{(E B)}$ Eclipsing binary star. ${ }^{(v)}$ Variable star. ${ }^{(a)}$ Values interpolated from Cox (2000).

clusters is very important not only to derive cluster distances and ages but also to discuss the stellar structure and evolution. To this end, in the very near future we shall study the formation and evolution of Be stars through the consideration of the frequency of $\mathrm{Be}$ stars relative to normal B stars with similar ages and masses. In fact, Be stars are main-sequence objects that probably formed with higher initial rotational velocities than B-normal. In our Galaxy, the Be phenomenon in massive stars often appears in the first part of the main sequence, while among less massive B-stars it appears in the second half of their main-sequence lifespan (Zorec et al. 2005). In contrast, massive Be stars in the Magellanic Clouds, and more particularly in the Small Magellanic Cloud, are more numerous because of the low metallicity (Maeder et al. 1999), and also they remain as such in the second part of the main sequence (Martayan et al. 2007). This difference in the presence of massive Be stars in different periods of their main-sequence life is due to the loss of angular momentum through stellar winds, which is stronger the higher the metallicity. Galactic open clusters in different chemical environments are thus optimal places to test the evolution of massive stars as rapid rotators. To this purpose, it is necessary to gather a high number of B and Be stars with reliable fundamental stellar parameters to make the sample statistically relevant. We conducted a spectrophotometric survey of roughly 110 early-type stars in five clusters located in the third and fourth quadrant of our Galaxy. Other galactic clusters in other galaxies, as well as in the Magellanic Clouds, will be studied in the same way in the near future.

As the $\operatorname{BCD}\left(\lambda_{1}, D\right)$ parameters are not affected by interstellar/circumstellar absorption, we were able to build accurate HR diagrams, derive cluster parameters, such as distances and ages, obtain physical properties of $\mathrm{B}$ and Be stars, and report new Be candidates as well as blue stragglers. We were also able to detect the overlapping of clusters in the sight direction and to discuss the stellar membership to clusters.

The results of the studied open clusters are summarized below:

Collinder 223: we report the following cluster parameters: $\overline{E(B-V)}=0.25 \pm 0.03 \mathrm{mag}$ and $\overline{\left(m_{\mathrm{v}}-M_{\mathrm{v}}\right)_{0}}=11.21 \pm 0.25$ $\left(\langle d\rangle=1746_{-186}^{+209}\right.$ pc $)$. We confirm that stars Nos. 2, 4, 29, and 80 are non-members (in agreement with the results given by Claria \& Lapasset 1991), while stars Nos. 5, 9, 21, and 35, as well as HD 305291 and HD 305296 are pnm. The age of the cluster still remains uncertain.

We have reported the spectral classification of seven stars for the first time and provide stellar parameters for all the sample. We discard Nos. 80 and 35 as blue stragglers since the former is a non-member and the latter is a pnm. However, we find that the Be star HD 305296 could be a real blue straggler object. We also suggest that stars Nos. 2, 4, 21, and 106 are Be candidates because of the presence of a second Balmer discontinuity in their spectra.

Hogg 16: here we clearly distinguish two population of stars with very different mean true distance moduli (Hogg 16a with $8.91 \pm 0.26 \mathrm{mag}$ and $\mathrm{Hogg} 16 \mathrm{~b} 12.51 \pm 0.38 \mathrm{mag}$ ), colour excesses $(0.26 \pm 0.03 \mathrm{mag}$ and $0.63 \pm 0.08 \mathrm{mag})$ and spectral types (B early-type stars and B late and A-type stars, respectively).

The more distant group then could be merged with Collinder 272. The age of the first group remains very uncertain while the age of the second group is between $10 \mathrm{Myr}$ and 16 Myr.

We have classified five stars for the first time. For the other stars of the sample, our classification suggests that the spectral types are earlier than those reported in previous works. 
Table 14. NGC 6025: Physical parameters and distance to stars.

\begin{tabular}{clccccccc}
\hline \hline $\begin{array}{c}\text { ID } \\
\begin{array}{c}\text { Feinstein } \\
\text { Kilambi }\end{array}\end{array}$ & $\begin{array}{l}\text { Other } \\
\text { designation }\end{array}$ & $\begin{array}{c}m_{\mathrm{v}} \\
{[\mathrm{mag}]}\end{array}$ & $E(B-V)$ & $\begin{array}{c}\left(m_{\mathrm{v}}-M_{\mathrm{v}}\right)_{0} \\
{[\mathrm{mag}]}\end{array}$ & $\begin{array}{c}d \\
{[\mathrm{pc}]}\end{array}$ & $\begin{array}{c}\log \mathcal{L} \\
{\left[\mathcal{L}_{\odot}\right]}\end{array}$ & $\begin{array}{c}M \\
{\left[M_{\odot}\right]}\end{array}$ & $\begin{array}{c}\text { Age } \\
{[\mathrm{Myr}]}\end{array}$ \\
\hline 01 & HD 143 448 & 7.30 & $0.35 \pm 0.01$ & $10.22 \pm 0.6$ & $1104 \pm 330$ & $4.76 \pm 0.26$ & $17.77 \pm 3.28$ & $7 \pm 2$ \\
02 & HD 143 449 & 8.11 & $0.36 \pm 0.01$ & $8.48 \pm 0.5$ & $496 \pm 108$ & $2.93 \pm 0.18$ & $5.56 \pm 0.61$ & $53 \pm 7$ \\
03 & HD 143413 & 8.42 & $0.31 \pm 0.01$ & $9.34 \pm 0.6$ & $737 \pm 190$ & $3.12 \pm 0.21$ & $6.77 \pm 1.46$ & $44 \pm 12$ \\
04 & CD-606021 & 9.73 & $0.26 \pm 0.01$ & $9.05 \pm 0.2$ & $647 \pm 63$ & $2.26 \pm 0.09$ & $3.47 \pm 0.15$ & $107 \pm 34$ \\
05 & CD-606007 & 10.23 & $0.24 \pm 0.02$ & $9.35 \pm 0.3$ & $742 \pm 111$ & $2.19 \pm 0.06$ & $3.19 \pm 0.18$ & $113 \pm 60$ \\
06 & HD 143 288 & 8.96 & $0.44 \pm 0.01$ & $8.06 \pm 0.3$ & $409 \pm 57$ & $2.53 \pm 0.12$ & $4.16 \pm 0.22$ & $53 \pm 28$ \\
07 & HD 143 340 & 8.05 & $0.41 \pm 0.01$ & $8.59 \pm 0.5$ & $523 \pm 134$ & $3.06 \pm 0.21$ & $6.53 \pm 1.36$ & $46 \pm 12$ \\
08 & CD-606015 & 8.86 & $0.27 \pm 0.01$ & $8.86 \pm 0.3$ & $592 \pm 87$ & $2.73 \pm 0.13$ & $4.80 \pm 0.31$ & $54 \pm 16$ \\
11 & HD 143 287 & 8.34 & $0.32 \pm 0.01$ & $7.69 \pm 0.2^{n m}$ & $345 \pm 32$ & $2.43 \pm 0.11$ & $3.88 \pm 0.21$ & $82 \pm 29$ \\
12 & HD 143 309 & 9.30 & $0.34 \pm 0.01$ & $10.44 \pm 0.5^{p n m}$ & $1227 \pm 291$ & $3.32 \pm 0.18$ & $7.54 \pm 1.19$ & $27 \pm 7$ \\
13 & CD-606011 & 9.62 & $0.13 \pm 0.01$ & $10.13 \pm 0.4$ & $1062 \pm 203$ & $2.71 \pm 0.17$ & $4.98 \pm 0.51$ & $59 \pm 13$ \\
14 & CD-606013 & 9.85 & $0.22 \pm 0.01$ & $9.90 \pm 0.3$ & $953 \pm 123$ & $2.64 \pm 0.12$ & $4.60 \pm 0.26$ & $49 \pm 19$ \\
15 & CD-606017 & 9.63 & $0.93 \pm 0.02$ & $7.57 \pm 0.2^{n m}$ & $327 \pm 24$ & $0.85 \pm 0.20$ & $1.51 \pm 0.24$ & $782 \pm 785$ \\
16 & CD-606027 & 9.88 & $0.40 \pm 0.01$ & $9.20 \pm 0.3$ & $692 \pm 109$ & $2.51 \pm 0.14$ & $4.17 \pm 0.31$ & $89 \pm 14$ \\
22 & HD 143 388 & 9.15 & $0.29 \pm 0.01$ & $9.71 \pm 0.4$ & $873 \pm 165$ & $2.99 \pm 0.18$ & $5.90 \pm 0.59$ & $39 \pm 10$ \\
23 & CD-606024 & 10.59 & $0.36 \pm 0.01$ & $10.92 \pm 0.4^{n m}$ & $1526 \pm 304$ & $2.93 \pm 0.20$ & $5.67 \pm 0.63$ & $46 \pm 10$ \\
24 & CPD-596562 & 10.06 & $0.38 \pm 0.01$ & $8.85 \pm 0.3$ & $588 \pm 83$ & $2.25 \pm 0.08$ & $3.45 \pm 0.17$ & $78 \pm 44$ \\
25 & HD 143412dd & 9.70 & $0.40 \pm 0.01$ & $8.72 \pm 0.3$ & $555 \pm 64$ & $2.38 \pm 0.08$ & $3.85 \pm 0.36$ & $48 \pm 32$ \\
26 & V 396 Nor & 9.31 & $0.36 \pm 0.03$ & $7.67 \pm 0.2^{n m}$ & $342 \pm 35$ & $1.79 \pm 0.13$ & $2.36 \pm 0.25$ & $181 \pm 174$ \\
43 & HD 143 123 & 9.34 & $0.44 \pm 0.01$ & $9.06 \pm 0.4$ & $648 \pm 107$ & $2.85 \pm 0.13$ & $5.35 \pm 0.62$ & $37 \pm 15$ \\
86 & CPD-606287 & 10.70 & $0.33 \pm 0.03$ & $8.38 \pm 0.5$ & $474 \pm 110$ & $1.46 \pm 0.20$ & $2.19 \pm 0.28$ & $264 \pm 174$ \\
90 & HD 143027 & 10.27 & $0.47 \pm 0.01$ & $10.23 \pm 0.5$ & $1110 \pm 248$ & $2.84 \pm 0.21$ & $5.48 \pm 0.78$ & $58 \pm 11$ \\
\hline
\end{tabular}

Notes. $m_{\mathrm{v}}$ values obtained from SIMBAD database. ${ }^{(d d)}$ Stars with double Balmer discontinuity. ${ }^{\left({ }^{(n)}\right)}$ Non-member stars. ${ }^{(p n m)}$ Probable non-member stars.

Among the known population of Be stars in Hogg 16, two of them (Nos. 3 and 68) show a second Balmer jump.

NGC 2645: this is a young open cluster $(<14$ Myr) located in a high reddening region, $\overline{E(B-V)}=0.58 \pm 0.05 \mathrm{mag}$, with a mean true distance modulus of $12.18 \pm 0.30 \mathrm{mag}$ $\left(d=2726_{-354}^{+407} \mathrm{pc}\right)$. We have determined the spectral classification of five stars for the first time and find a new Be field candidate, star No. 51. We show that the star Nos. 1, 51, 55, 56, 72, and HD 74180 are non-members.

NGC 3114: stars of this cluster are characterized by an anomalous colour gradient. To explain this result, we considered several possible scenarios and concluded that the Be phenomenon and binarity could account for this anomaly. The mean cluster parameters determined in this work are: $\overline{E(B-V)}=0.10 \pm 0.01 \mathrm{mag}$ and $\overline{\left(m_{\mathrm{v}}-M_{\mathrm{v}}\right)_{0}}=9.20 \pm$ $0.15 \mathrm{mag}$ (which corresponds to a $\langle d\rangle=694_{-46}^{+49} \mathrm{pc}$ ). However, owing to the anomalies found in the colour gradients, the distance could be overestimated. On the basis of our criterion, we find that stars Nos. 4, 33, 66, 105, 132, and 147 are pnm and Nos. 9, 100, 102, and 141 are non-members. We are not able to define the cluster age since the region seems to have a mixture of stars with different ages.

We have classified four stars for the first time. The determined spectral types for most of the stars of our sample agree with previous determinations, where possible discrepancies are not higher than two sub-spectral types. In our sample, we distinguish nine stars showing the second Balmer discontinuity, which are then considered Be candidates, namely: Nos. 3, 4, 6, 11, 79, 91, 126, 132, and HD 304831. Particularly, stars Nos. 3, 4, and 91, which show the second Balmer jump, were reported by González \& Lapasset (2001) as line-emission stars. This stars are therefore considered to be confirmed Be stars.
NGC 6025: this is a young open cluster (between $40 \mathrm{Myr}$ and $69 \mathrm{Myr})$ with a moderated reddening $(\overline{E(B-V)}=$ $0.34 \pm 0.02 \mathrm{mag})$ located at a true mean distance modulus of $9.25 \pm 0.17 \mathrm{mag}\left(\langle d\rangle=709_{-53}^{+57} \mathrm{pc}\right)$. Our HR diagram shows a lack of stars with $M_{\text {bol }}$ between 0 and 1 mag, but to confirm the existence of the gap (reported by Mermilliod 1981; Kilambi 1975) we need more observations. According to our criterion of membership determination, we consider that stars Nos. 11, 15, 23, and 26 are non-members and the star No. 12 is a pnm.

Stars Nos. 3 and 25 are Be candidates since they show a second Balmer discontinuity.

Finally, we want to stress the presence of detached stars from the main sequence towards lower temperatures or high luminosities in the HR diagrams of Collinder 223 and NGC 3114. This bump is originated by late B-type stars with $\mathrm{M}_{\mathrm{bol}}$ between -1 mag and $1 \mathrm{mag}$. The origin of this bump could be related to the underestimation of the reddening produced by the anomalous spectral gradient of the stars, rapid stellar rotation that induces the GDE, binary-related effects, and/or tidal interactions with nearby clusters. Hence, further investigations of the incidence of binarity in clusters and the effect of the stellar rotation on the emerging radiation should be carried out.

Acknowledgements. We would like to thank our anonymous referee for his/her valuable comments and suggestions. This research has made use of the SIMBAD database, operated at CDS, Strasbourg, France, and WEBDA database, operated at the Department of Theoretical Physics and Astrophysics of the Masaryk University. L.C. thanks financial support from the Agencia de Promoción Científica y Tecnológica (Préstamo BID PICT 2011/0885), CONICET (PIP 0300), and the Universidad Nacional de La Plata (Programa de Incentivos G11/109), Argentina. 


\section{References}

Ahumada, J., \& Lapasset, E. 1995, A\&AS, 109, 375

Aidelman, Y., Cidale, L. S., Zorec, J., \& Arias, M. L. 2012, A\&A, 544, A64

Amieux, G., \& Burnage, R. 1981, A\&AS, 44, 101

Barbier, D., \& Chalonge, D. 1941, Annales d'Astrophysique, 4, 30

Becker, W., \& Fenkart, R. 1971, A\&AS, 4, 241

Bidelman, W. P. 1951, ApJ, 113, 304

Boehm-Vitense, E. 1982, ApJ, 255, 191

Bowman, A. W., \& Azzalini, A. 1997, Applied smoothing techniques for data analysis: the kernel approach with S-plus illustrations, Oxford statistical science series No. 18 (Clarendon Press)

Bukowiecki, Ł., Maciejewski, G., Konorski, P., \& Strobel, A. 2011, Acta Astron., 61, 231

Buscombe, W. 1969, MNRAS, 144, 31

Bychkov, V. D., Bychkova, L. V., \& Madej, J. 2009, MNRAS, 394, 1338

Carraro, G., \& Patat, F. 2001, A\&A, 379, 136

Chalonge, D., \& Divan, L. 1952, Annales d'Astrophysique, 15, 201

Chalonge, D., \& Divan, L. 1977, A\&A, 55, 117

Cidale, L. S., Arias, M. L., Torres, A. F., et al. 2007, A\&A, 468, 263

Claria, J. J., \& Lapasset, E. 1991, PASP, 103, 998

Collins, II, G. W., Truax, R. J., \& Cranmer, S. R. 1991, ApJS, 77, 541

Cox, A. N. 2000, Allen's astrophysical quantities, 4th edn. (New York: AIP Press and Springer)

Divan, L., Zorec, J., \& Briot, D. 1982, in Be Stars, eds. M. Jaschek, \& H.-G. Groth, IAU Symp., 98, 53

Ekström, S., Georgy, C., Eggenberger, P., et al. 2012, A\&A, 537, A146

Feast, M. W., Stoy, R. H., Thackeray, A. D., \& Wesselink, A. J. 1961, MNRAS, 122,239

Feinstein, A. 1971, PASP, 83, 800

Fenkart, R. P., \& Binggeli, B. 1979, A\&AS, 35, 271

Fenkart, R. P., Binggeli, B., Good, D., et al. 1977, A\&AS, 30, 307

Fitzgerald, M. P., Boudreault, R., Fich, M., Luiken, M., \& Witt, A. N. 1979a, A\&AS, 37, 351

Fitzgerald, M. P., Luiken, M., Maitzen, H. M., \& Moffat, A. F. J. 1979b, A\&AS, 37,345

Flower, P. J. 1996, ApJ, 469, 355

Forbes, D., \& Short, S. 1994, AJ, 108, 594

Frémat, Y., Zorec, J., Hubert, A., \& Floquet, M. 2005, A\&A, 440, 305

Frye, R. L., MacConnell, D. J., \& Humpherys, R. M. 1970, PASP, 82, 1360

Garrison, R. F., Hiltner, W. A., \& Schild, R. E. 1977, ApJS, 35, 111

González, J. F., \& Lapasset, E. 2001, AJ, 121, 2657

González, J. F., \& Levato, H. 2006, A\&A, 448, 283

Graham, J. A. 1970, AJ, 75, 703

Gray, R. O., \& Garrison, R. F. 1989, ApJS, 70, 623

Grosso, M., \& Levato, H. 2011, Rev. Mex. Astron. Astrofis., 47, 255

Hamdy, M. A., Abo Elazm, M. S., \& Saad, S. M. 1993, Ap\&SS, 203, 53

Henize, K. G. 1976, ApJS, 30, 491

Hogg, A. R. 1953, MNRAS, 113, 746

Hogg, A. R. 1965, PASP, 77, 440

Houk, N. 1978, Michigan catalogue of two-dimensional spectral types for the HD stars

Houk, N., \& Cowley, A. P. 1975, University of Michigan Catalogue of twodimensional spectral types for the HD stars, Vol. I, Declinations -90_ to $53 \_f 0$

Humphreys, R. M. 1978, ApJS, 38, 309

Iriarte, B. 1965, Boletin de los Observatorios Tonantzintla y Tacubaya, 4, 33

Jankowitz, N. E., \& McCosh, C. J. 1962, Mon. Notes. Astr. Soc. South Africa, 22,18

Jaschek, M., \& Egret, D. 1982, in Be Stars, eds. M. Jaschek, \& H.-G. Groth, IAU Symp., 98, 261

Kharchenko, N. V., Piskunov, A. E., Röser, S., Schilbach, E., \& Scholz, R. 2005, A\&A, 438, 1163

Kharchenko, N. V., Berczik, P., Petrov, M. I., et al. 2009a, A\&A, 495, 807

Kharchenko, N. V., Piskunov, A. E., Röser, S., et al. 2009b, A\&A, 504, 681

Kilambi, G. C. 1975, PASP, 87, 975

Kopchev, V., Nedialkov, P., \& Petrov, G. 2006, Bulg. J. Phys., 33, 68

Lapasset, E., Claria, J. J., \& Minniti, D. 1991, Information Bulletin on Variable Stars, 3594, 1
Leone, F., \& Catalano, F. A. 1991, A\&A, 242, 199

Levato, H., \& Malaroda, S. 1975, AJ, 80, 807

Lindoff, U. 1968, Arkiv for Astronomi, 5, 1

Loktin, A. V., \& Beshenov, G. V. 2001, Astron. Lett., 27, 386

Lunt, J. 1918, ApJ, 48, 261

Lyngå, G. 1962, Arkiv for Astronomi, 3, 65

Mackey, A. D., \& Broby Nielsen, P. 2007, MNRAS, 379, 151

Maeder, A., \& Peytremann, E. 1970, A\&A, 7, 120

Maeder, A., Grebel, E. K., \& Mermilliod, J.-C. 1999, A\&A, 346, 459

Maitzen, H. M., Weiss, W. W., \& Schneider, H. 1988, A\&AS, 75, 391

Malaroda, S. 1975, AJ, 80, 637

Malysheva, L. K. 1997, Astron. Lett., 23, 585

Martayan, C., Frémat, Y., Hubert, A.-M., et al. 2007, A\&A, 462, 683

Mermilliod, J. C. 1981, A\&AS, 44, 467

Mermilliod, J. C. 1982a, A\&A, 109, 48

Mermilliod, J.-C. 1982b, A\&A, 109, 37

Merrill, P. W., \& Burwell, C. G. 1933, ApJ, 78, 87

Mersch, G., \& Heck, A. 1980, A\&A, 85, 93

Meynet, G., Mermilliod, J.-C., \& Maeder, A. 1993, A\&AS, 98, 477

Moffat, A. F. J., \& Fitzgerald, M. P. 1977, A\&A, 54, 263

Moffat, A. F. J., \& Vogt, N. 1973a, A\&A, 23, 317

Moffat, A. F. J., \& Vogt, N. 1973b, A\&AS, 10, 135

Moore, J. H., Paddock, G. F., Wilson, R. E., \& Campbell, W. W. 1914, Lick Observatory Bulletin, 8, 71

Morris, P. M. 1961, MNRAS, 122, 325

Moujtahid, A., Zorec, J., Hubert, A. M., Garcia, A., \& Burki, G. 1998, A\&AS, 129,289

Moujtahid, A., Zorec, J., \& Hubert, A. M. 1999, A\&A, 349, 151

Moujtahid, A., Hubert, A. M., Zorec, J., et al. 2000, in The Be Phenomenon in Early-Type Stars, eds. M. A. Smith, H. F. Henrichs, \& J. Fabregat, ASP Conf. Ser., 214, IAU Colloq. 175, 514

Nesterov, V. V., Kuzmin, A. V., Ashimbaeva, N. T., et al. 1995, A\&AS, 110, 367

Nicolet, B. 1981, A\&A, 104, 185

Palate, M., Rauw, G., Koenigsberger, G., \& Moreno, E. 2013, A\&A, 552, A39

Pandey, A. K., Sandhu, T. S., Sagar, R., \& Battinelli, P. 2010, MNRAS, 403, 1491

Paunzen, E., \& Netopil, M. 2006, MNRAS, 371, 1641

Piskunov, A. E., Schilbach, E., Kharchenko, N. V., Röser, S., \& Scholz, R.-D. 2008, A\&A, 477, 165

Pišmiš, P. 1959, Boletin de los Observatorios Tonantzintla y Tacubaya, 2, 37

Quiroga, C., Torres, A. F., \& Cidale, L. S. 2010, A\&A, 521, A75

Rastorguev, A. S., Glushkova, E. V., Dambis, A. K., \& Zabolotskikh, M. V. 1999 , Astron. Lett., 25, 595

Renson, P., \& Manfroid, J. 2009, A\&A, 498, 961

Robichon, N., Arenou, F., Mermilliod, J.-C., \& Turon, C. 1999, A\&A, 345, 471

Sagar, R., \& Sharples, R. M. 1991, A\&AS, 88, 47

Santiago, B., Kerber, L., Castro, R., \& de Grijs, R. 2002, MNRAS, 336, 139

Santos-Silva, T., \& Gregorio-Hetem, J. 2012, A\&A, 547, A107

Schmidt, E. G. 1982, PASP, 94, 232

Schneider, H., \& Weiss, W. W. 1988, A\&AS, 75, 353

Smart, W. M. 1958, Combination of observations (Cambridge: Cambridge University Press)

Stateva, I., Iliev, I. K., \& Budaj, J. 2012, MNRAS, 420, 1207

Steemers, W. J. G., \& van Genderen, A. M. 1986, A\&A, 154, 308

Tadross, A. L. 2001, New Astron., 6, 293

Tadross, A. L. 2004, Ch. J. Astron. Astrophys., 4, 67

Torres, A. F., \& Cidale, L. S. 2007, in Active OB-Stars: Laboratories for Stellare and Circumstellar Physics, eds. A. T. Okazaki, S. P. Owocki, \& S. Stefl, ASP Conf. Ser., 361, 509

van Leeuwen, F., van Genderen, A. M., \& Zegelaar, I. 1998, A\&AS, 128, 117

Vazquez, R. A., \& Feinstein, A. 1991, A\&AS, 90, 317

Vazquez, R. A., Baume, G., Feinstein, A., \& Prado, P. 1997, A\&AS, 124, 13

Vogt, N., \& Moffat, A. F. J. 1973, A\&AS, 9, 97

von Zeipel, H. 1924, MNRAS, 84, 684

Wade, B. R., \& Smith, L. F. 1985, MNRAS, 212, 77

Waelkens, C., \& Rufener, F. 1985, A\&A, 152, 6

Zorec, J., Frémat, Y., \& Cidale, L. 2005, A\&A, 441, 235 